\title{
4-Engel groups are locally nilpotent
}

\author{
George Havas \\ ARC Centre for Complex Systems \\ School of Information Technology and Electrical Engineering \\ The University of Queensland, Queensland 4072, Australia \\ havas@itee.uq.edu.au \\ M. R. Vaughan-Lee \\ Christ Church \\ Oxford OX1 1DP \\ England \\ michael.vaughan-lee@christ-church.oxford.ac.uk
}

\begin{abstract}
Questions about nilpotency of groups satisfying Engel conditions have been considered since 1936, when Zorn proved that finite Engel groups are nilpotent. We prove that 4-Engel groups are locally nilpotent. Our proof makes substantial use of both hand and machine calculations.

Keywords: Engel groups; nilpotent groups.

AMS Mathematics Subject Classification: primary 20F05, 20F45; secondary 20-04.
\end{abstract}

\section{Introduction}

In this article we prove:

Theorem 1. 4-Engel groups are locally nilpotent.

A group $G$ is said to be an $n$-Engel group if

$$
[x, \underbrace{y, y, \ldots, y}_{n}]=1
$$

for all $x, y \in G$. (Here $[x, y]$ denotes $x^{-1} y^{-1} x y$, and $[x, y, \ldots, y]$ denotes the left-normed commutator $[[\ldots[x, y], \ldots], y]$.$) Burnside [4]$ already considered properties of 2-Engel groups in 1903. The structure of 2-Engel groups is straightforward, as they are known to be nilpotent of class at most 3 - see Levi [12]. Heineken [9] proved that 3-Engel groups are locally nilpotent. He showed further that a 3-Engel group is nilpotent of 
class at most 4 if it has no elements of order 2 or 5 . On the other hand there are 3-Engel 2-groups and 3-Engel 5-groups which are not nilpotent (see Gruenberg [7] and Bachmuth and Mochizuki [2]). Kappe and Kappe [11] proved that a group is a 3-Engel group if and only if the normal closure of every element is nilpotent of class at most 2 .

Much is known about the structure of locally nilpotent 4-Engel groups. In particular Traustason [20] has proved that if $G$ is a locally nilpotent 4-Engel group, and if $g \in G$, then the normal closure of $g$ in $G$ is nilpotent of class at most 4. Traustason [19] showed that if 4-Engel groups of exponent $p$ are locally finite, then 4-Engel $p$-groups are locally finite. It follows immediately that 4-Engel 2-groups and 4-Engel 3-groups are locally finite. Vaughan-Lee [22] proved that 4-Engel groups of exponent 5 are locally finite, and it follows from this and Traustason's work that 4-Engel 5-groups are locally finite. Newman and Vaughan-Lee [13] computed the orders of the free 4-Engel groups of exponent 5 .

Traustason [19] has shown that locally nilpotent 4-Engel groups without elements of order 2 or 3 or 5 are nilpotent of class at most 7. Abdollahi and Traustason [1] showed that locally nilpotent 4-Engel groups without elements of order 2 and 5 are soluble, though they need not be nilpotent. But there are examples of 4-Engel 2-groups and 4-Engel 5-groups which are not soluble (see [2] and [16]).

Recently Traustason [21] proved that 2-generator, 4-Engel groups are nilpotent. This important result encouraged us to look again at the question of local nilpotence in general 4-Engel groups. Our approach follows the same line of argument as was used in [22] to prove that 4-Engel groups of exponent 5 are locally finite, though the details here differ significantly from those in [22]. Our proof of Theorem 1 heavily relies on Traustason's result that 2-generator, 4-Engel groups are nilpotent, and makes use of the Knuth-Bendix procedure to show that certain 3-generator, 4-Engel groups are nilpotent.

Theorem 1, together with the results on locally nilpotent 4-Engel groups mentioned above, gives the following corollary:

Corollary 2. If $G$ is an m-generator, 4-Engel group then $G$ is nilpotent of class at most $4 m$, and if $G$ has no elements of order 2,3 or 5 then $G$ is nilpotent of class at most 7 .

Combined with the detailed results about nilpotent quotients of Engel groups given by Nickel [15], Theorem 1 gives the following two corollaries.

Corollary 3. The free, 2-generator, 4-Engel group is torsion free, has nilpotency class 6 and Hirsch length 11.

Corollary 4. The free, 3-generator, 4-Engel group has nilpotency class 9 and Hirsch length 88. Its torsion subgroup is isomorphic to $C_{5}^{44} \times C_{10}^{5} \times C_{30}^{4} \times C_{60}^{4}$.

Corollary 3 is already a consequence of Traustason's result [21]. Further information about these free Engel groups may be read from their polycyclic presentations. In general, using Lie methods, we can show that the torsion subgroup of a free 4-Engel group is a $\{2,3,5\}$-group. 


\section{The Hirsch-Plotkin radical}

If $G$ is any group then the Hirsch-Plotkin radical of $G$ is the product of the locally nilpotent normal subgroups of $G$. If $G$ is a 4-Engel group, and if $G$ has HirschPlotkin radical $R$, then the Hirsch-Plotkin radical of $G / R$ is trivial. (See Lemma 1.1 of [21] for a short proof of this result.) In particular, the centre of $G / R$ is trivial. Traustason's theorem that 2-generator, 4-Engel groups are nilpotent implies that the torsion elements in a 4-Engel group form a subgroup which is a direct product of $p$ groups. And so the theorems that 4-Engel $p$-groups are locally nilpotent for $p=2,3,5$ imply that if $G$ is a 4 -Engel group then $G / R$ has no elements of order 2 or 3 or 5 . We will make repeated use of these facts in the following way. In Section 4 , for example, we consider a 4 -Engel group $G$ generated by three elements $u, v, w$ satisfying the relations

$$
[u, v]=[w, u, u, u]=[w, u, u, w]=[w, u, w, w]=[w, v, v]=[w, v, w]=1 .
$$

Our aim is to prove that $G$ is nilpotent. If we pass to the quotient $G / R$, where $R$ is the Hirsch-Plotkin radical of $G$, then $G / R$ is a three generator group with generators $\bar{u}=u R, \bar{v}=v R, \bar{w}=w R$ satisfying the relations

$$
[\bar{u}, \bar{v}]=[\bar{w}, \bar{u}, \bar{u}, \bar{u}]=[\bar{w}, \bar{u}, \bar{u}, \bar{w}]=[\bar{w}, \bar{u}, \bar{w}, \bar{w}]=[\bar{w}, \bar{v}, \bar{v}]=[\bar{w}, \bar{v}, \bar{w}]=1 .
$$

The remarks above imply that $G / R$ has trivial centre, and that $G / R$ has no elements of order 2 or 3 or 5 . If (with these assumptions) we can show that $G / R$ is nilpotent, then it follows that $R=G$ and that $G$ is nilpotent. In applications of this argument we will avoid complicating the notation by simply assuming that the original group $G$ has trivial centre and has no elements of order 2 or 3 or 5 .

\section{An overview of the machine computations}

Our proof makes substantial use of both hand and machine calculations. In this section we provide an overview of our machine computations, including an indication of how specific computations were motivated. We also provide some representative timings.

We started by aiming to extend the results of Vaughan-Lee [22], which showed that 4-Engel 5-groups are locally finite, to $p$-groups for other primes. Vaughan-Lee's proof also relies on both hand and machine calculations. In that case the machine work involved use of the $p$-quotient algorithm and of coset enumeration. Indeed we were able to prove similar results for primes $p$ up to 31. The nature of the machine computations was similar, though the details varied. In particular we found that we needed to impose more instances of Engel relations for higher primes.

These results provided solid justification for believing that 4-Engel $p$-groups are locally finite for general $p$, so our aim became the full generalization of that result. To prove the more general result, namely local nilpotence for 4-Engel groups, the previous machine computation tools were no longer directly adequate. Where Vaughan-Lee previously used the $p$-quotient algorithm to prove properties of $p$-groups, we use a nilpotent quotient algorithm to prove properties of more general nilpotent groups. Where Vaughan-Lee previously used coset enumeration to prove finiteness of finitely 
presented groups, we use the Knuth-Bendix procedure to prove nilpotence. We discuss application of the nilpotent quotient algorithm in the remainder of this section and we describe our use of the Knuth-Bendix procedure in the next section.

We use the nilpotent quotient algorithm [14] (as incorporated in the computer algebra systems GAP [5] and MAGMA [3]) to obtain information about the nilpotent quotients of finitely generated 4-Engel groups. Note that the nilpotent quotient algorithm can find nilpotent quotients of a specified finitely presented group, but it cannot show that the finitely presented group is nilpotent. Application of the nilpotent quotient algorithm to finitely presented groups is straightforward. Also, implementations of the nilpotent quotient algorithm allow ready imposition of Engel laws on finitely presented groups, see [15]. The nilpotent quotient algorithm produces as output a polycyclic presentation (PCP) of a nilpotent quotient of the finitely presented group revealing properties of the nilpotent quotient. For example, the nilpotent quotient algorithm shows that a nilpotent quotient of a 3-generator, 4-Engel group satisfying the relations (1) above has class at most 4.

Using the latest version of the nilpotent quotient algorithm, Version 2.0, we can compute nilpotent quotients of 2- and 3-generator, 4-Engel groups quite quickly. On a Pentium III machine with a $500 \mathrm{MHz}$ cpu running linux (not a fast machine) the largest nilpotent quotient of the free, 2-generator, 4-Engel group (as in Corollary 3) can be found in $0.06 \mathrm{cpu}$ seconds; the largest nilpotent quotient of the free, 3-generator, 4-Engel group (as in Corollary 4) can be found in $3578 \mathrm{cpu}$ seconds (less than one cpu hour); and the largest nilpotent quotient of a 3-generator, 4-Engel group satisfying the relations (1) can be found in $0.02 \mathrm{cpu}$ seconds.

One application of the nilpotent quotient algorithm that we use frequently is the following. Suppose we are trying to prove that a certain finitely generated 4-Engel group $G$ is nilpotent. And suppose that we have two elements $a, b \in G$ and have managed to establish (for example) that $\left[a^{3}, b\right]=1$. We know from Traustason's result that the subgroup generated by $a, b$ is nilpotent. And the nilpotent quotient algorithm tells us that if $a, b$ generate a nilpotent 4-Engel group satisfying $\left[a^{3}, b\right]=1$ then $[a, b]^{9}=1$. So we have $[a, b]^{9}=1$ in $G$. And if we are making the assumption (as outlined in Section 2) that $G$ has no elements of order 3 , then we have $[a, b]=1$.

\section{The Knuth-Bendix procedure}

The use of the Knuth-Bendix process as a tool for group theory was pioneered by Sims. The basic procedure (in its application to groups) is described in [6] and (in great detail) in [18]. A major success of the Knuth-Bendix procedure in group theory was its use by Sims [17] to verify nilpotency of a finitely presented group. The nilpotent quotient algorithm was used initially to construct a polycyclic presentation for the nilpotent quotient $Q$ of the finitely presented group $G$. Then, using some relations of this presentation as part of an initial set of rewrite rules, and a special term-ordering, Sims was able to develop an effective algorithm for verifying the triviality of the kernel of the quotient $Q$.

We follow this kind of approach to address one particular group which plays a 
crucial role in our overall proof. This group, which we call $T$, is a 3-generator 4-Engel group which has nilpotency conditions applying to the subgroups generated by each pair of its given generators. One pair of generators commute, another pair generate a subgroup with class 2 , and the remaining pair generate a subgroup with class 3 . We start with the following presentation for $T$ :

$$
\langle u, v, w|[u, v],[w, u, u, u],[w, u, u, w],[w, u, w, w],[w, v, v],[w, v, w], 4 \text {-Engel }\rangle .
$$

$T$ arises as a preimage of a number of groups which we need to prove nilpotent. The proof that those groups are nilpotent follows directly from the fact $T$ is nilpotent. Here we show how the nilpotency of $T$ may be proved.

Knuth-Bendix implementations well-suited to group theory which are available include RKBP by Sims (see the Acknowledgements) and KBMAG by Holt (see [10]) which is available in GAP and MAGMA. These Knuth-Bendix packages offer a bewilderingly large choice of parameter settings. Very important is the choice of ordering for words in the generators. Both of these packages may be used in proving the requisite result about $T$. Since RKBP includes a facility for automatically imposing Engel laws we commenced with it.

The final result is that the Knuth-Bendix procedure (together with "hand" work) shows that the group $T$ is nilpotent. The nilpotent quotient algorithm then shows that it is nilpotent of class 4 . The Knuth-Bendix details follow.

A simple-minded approach to solving this problem is as follows. Use the nilpotent quotient algorithm to find a PCP for the largest nilpotent quotient. We find that the largest nilpotent quotient of $T$ has class 4, and has a PCP on 10 generators

$$
u, v, w,[w, u],[w, v],[w, u, u],[w, u, w],[w, v, u],[w, v, u, u],[w, v, u, w] .
$$

Denote these PC generators by $x_{1}, x_{2}, \ldots, x_{10}$. Define an input group for Knuth-Bendix on generators $x_{1}, x_{2}, \ldots, x_{10}$, with relations $x_{4}=\left[x_{3}, x_{1}\right], x_{5}=\left[x_{3}, x_{2}\right], x_{6}=\left[x_{4}, x_{1}\right]$, $x_{7}=\left[x_{4}, x_{3}\right], x_{8}=\left[x_{5}, x_{1}\right], x_{9}=\left[x_{8}, x_{1}\right], x_{10}=\left[x_{8}, x_{3}\right]$ giving the "definitions" of $x_{4}, x_{5}, \ldots, x_{10}$ and relations $\left[x_{2}, x_{1}\right]=\left[x_{5}, x_{2}\right]=\left[x_{5}, x_{3}\right]=\left[x_{6}, x_{1}\right]=\left[x_{6}, x_{3}\right]=\left[x_{7}, x_{3}\right]$ $=1$ corresponding to defining relations of $T$. Then run the Knuth-Bendix process using a wreath-product ordering with this input group, adding sufficient instances of the 4-Engel law till the process terminates with a confluent presentation for the group. The wreath-product ordering means that the Knuth-Bendix procedure attempts to rewrite words on these generators as normal words $x_{1}^{\alpha_{1}} x_{2}^{\alpha_{2}} \ldots x_{10}^{\alpha_{10}}$.

This approach works well with easier problems about 3-generator groups. It readily shows the nilpotency of any 3-generator 4-Engel group in which two pairs of generators commute and the remaining pair generate a subgroup with class 3 . The largest such group is torsionfree and has class 3 with six generators in its PCP. With more effort, but still easily, it shows the nilpotency of any 3-generator 4-Engel group in which one pair of generators commute and the other two pairs generate a subgroup with class 2. The largest such group has class 4 and seven generators in its PCP, with its last generator having order 2 .

On a Sun Sparcv9 machine with a $750 \mathrm{MHz}$ cpu running SunOS we can use RKBP version 1.51 this way to obtain a confluent presentation for $\langle u, v, w|[u, v]=[v, w]=$ 
$[w, u, u, u]=[w, u, u, w]=[w, u, w, w]=1,4$-Engel $\rangle$ in 13.3 cpu seconds. For $\langle u, v, w|$ $[u, v]=[w, u, u]=[w, u, w]=[w, v, v]=[w, v, w]=1,4$-Engel $\rangle$ we can obtain a confluent presentation in $2264 \mathrm{cpu}$ seconds.

We have not been able to make this approach work directly for $T$. When we start this way we find that the Knuth-Bendix procedure finds many thousands of consequences of the defining relations but does not terminate even when given large amounts of time and space. Various unsuccessful attempts ran for several cpu days and used up to a gigabyte of memory. Instead, our proof relies on some hand calculations and on our inspecting an incomplete Knuth-Bendix computation and deducing useful consequences by hand.

As we did this we discovered two important facts. First, it could be useful to introduce additional, redundant generators. Second, even though the wreath-product ordering implies that the simple approach should lead (eventually) to a finite confluent presentation, we found we could make more rapid progress using lenlex ordering. (We point out that the reduction to just the nilpotency problem for the group $T$ came after we had solved similar problems for various other groups. Also, the final proof for $T$, which requires only one Knuth-Bendix run, was developed after we had done very many other runs.)

We now describe how the fact that $T$ is nilpotent can be proved. Supplementary materials including details of input and output files which demonstrate this are available at our web sites [8] where we also provide a copy of the Knuth-Bendix program RKBP version 1.51 .

We start by deducing some additional relations which hold in $T$. This provides a small, sufficient set of relations for our further computations. (We have not tried to make this set of relations minimal.)

The defining relations imply that $\langle u, w\rangle$ is nilpotent of class 3 , and so in addition to the relations above we also obtain

$$
\left[x_{6}, x_{4}\right]=\left[x_{7}, x_{1}\right]=\left[x_{7}, x_{4}\right]=\left[x_{7}, x_{6}\right]=1 .
$$

Since $v$ commutes with $[w, v]$ and with $u$ we see that $[w, v, u, v]=[w, v, u, u, v]=1$, giving $\left[x_{8}, x_{2}\right]=\left[x_{9}, x_{2}\right]=1$.

Now we let $y=u w$ and consider the subgroup generated by $v$ and $y$. We have $[y, v]=[w, v]$ and hence $[y, v, v]=1$. Since $\langle y, v\rangle$ is a nilpotent 4-Engel group the relation $[y, v, v]=1$ implies that $[y, v, y, v]=1$. This gives

$$
1=[y, v, y, v]=[[w, v, u][w, v, u, w], v]=[w, v, u, w, v]
$$

since (as we observed above) $[w, v, u, v]=1$. The Jacobi-Witt identity

$$
\left[x, y^{-1}, z\right]^{y}\left[y, z^{-1}, x\right]^{z}\left[z, x^{-1}, y\right]^{x}=1
$$

gives

$$
[[w, v, u], w, v]^{w^{-1}}\left[w^{-1}, v^{-1},[w, v, u]\right]^{v}\left[v,[w, v, u]^{-1}, w^{-1}\right]^{[w, v, u]}=1,
$$

and this together with $[w, v, u, v]=1$ gives $[w, v, u,[w, v]]=1$. Since $[w, v]$ commutes with $w$ we also have $[w, v, u, w,[w, v]]=1$. The three relations

$$
[w, v, u, w, v]=[w, v, u,[w, v]]=[w, v, u, w,[w, v]]=1
$$


give that $\left[x_{10}, x_{2}\right]=\left[x_{8}, x_{5}\right]=\left[x_{10}, x_{5}\right]=1$.

If we consider the nilpotent 2-generator subgroup $\langle u,[w, v]\rangle$, then the relation $[w, v, u,[w, v]]=1$ implies that

$$
[w, v, u, u,[w, v]]=[w, v, u, u,[w, v, u]]=1,
$$

so $\left[x_{9}, x_{5}\right]=\left[x_{9}, x_{8}\right]=1$.

Using the Jacobi-Witt identity once more, we obtain

$$
\left[w, u^{-1}, v^{-1}\right]^{u}[u, v, w]^{v^{-1}}\left[v^{-1}, w^{-1}, u\right]^{w}=1 .
$$

Now

$$
\left[w, u^{-1}, v^{-1}\right]^{u}=\left[\left[w, u^{-1}\right]^{u}, v^{-1}\right]=\left[[w, u]^{-1}, v^{-1}\right],
$$

and $\left[v^{-1}, w^{-1}\right]=[w, v]^{-1}$, so that in the nilpotent group $\langle u,[w, v]\rangle$ described above we have

$$
\left[v^{-1}, w^{-1}, u\right]=[w, v, u]^{-1} \text {. }
$$

So we obtain

$$
\left[[w, u]^{-1}, v^{-1}\right]=[w, v, u]^{w}
$$

which gives the relation

$$
\left[x_{4}^{-1}, x_{2}^{-1}\right]=x_{8} x_{10} .
$$

We have already observed that $x_{8}$ and $x_{10}$ commute with $x_{2}$, and so we have $\left[x_{4}^{-1}, x_{2}^{-1}, x_{2}\right]=$ 1. In the nilpotent 4-Engel group $\left\langle x_{2}, x_{4}\right\rangle$ this implies the relation $\left[x_{4}, x_{2}, x_{2}\right]=1$, or $[w, u, v, v]=1$, and also $\left[x_{4}^{-1}, x_{2}, x_{2}\right]=\left[x_{4}, x_{2}^{-1}, x_{2}\right]=1$.

The relation

$$
\left[[w, v], w^{-1}, u\right]^{w}\left[w, u^{-1},[w, v]\right]^{u}\left[u,[w, v]^{-1}, w\right]^{[w, v]}=1
$$

gives $\left[x_{5} x_{8}, x_{4}^{-1}\right]=x_{10}$.

Finally, we have $[w, u v]=[w, v][w, u][w, u, v],[w, v u]=[w, u][w, v][w, v, u]$. Since $u v=v u$ this gives

$$
[w, v][w, u][w, u, v]=[w, u][w, v][w, v, u]
$$

which gives

$$
\left[x_{4}, x_{2}\right]=[w, u, v]=[[w, u],[w, v]][w, v, u]=\left[x_{4}, x_{5}\right] x_{8} .
$$

Since $x_{8}=[w, v, u]$ commutes with $v,[w, u, v, v]=1$ gives $\left[x_{4}, x_{5}, x_{2}\right]=1$.

In order to show that $T$ is nilpotent it suffices to have relations which imply that $\left[x_{9}, x_{1}\right],\left[x_{9}, x_{2}\right],\left[x_{9}, x_{3}\right],\left[x_{10}, x_{1}\right],\left[x_{10}, x_{2}\right]$ and $\left[x_{10}, x_{3}\right]$ are trivial. (Above we have already observed that $\left[x_{10}, x_{2}\right]=1$.) Finding relations involving $\left[x_{9}, x_{1}\right]$ turned out to be the most difficult of these. To obtain sufficient information about the commutator $\left[x_{9}, x_{1}\right]$ using the Knuth-Bendix process we found it useful to introduce an extra generator $x_{11}=\left[x_{9}, x_{1}\right]$.

We now commence a Knuth-Bendix run using lenlex ordering with all of these above relations defining our input group. The commands that we use with rkbp are: 
(i) input which reads in a new problem;

(ii) $\mathrm{kb}$ which iterates overlap formation a specified number of times, using overlaps of limited length, saving rules with bounded left and right hand sides;

(iii) add_engel which adds instances of the $n$-th Engel relation (for us $n=4$ ), evaluated for all pairs of words of bounded length containing specified PCP generators and inverses; and

(iv) rewrite_words which rewrites individual words using the current rewriting system.

Thus, we input the above defining relations for $T$ and execute kb 1021010 which does two iterations of overlap formation, using overlaps with lengths up to 10, saving rules with left and right hand sides with lengths up to 10. Using RKBP version 1.51 we obtain 4085 rules. Next we incorporate instances of the 4-Engel identity by executing add_engel 422051010 which adds the instances of the 4-th Engel relation evaluated for all pairs of words with length up to 2 containing $x_{1}, x_{1}^{-1}, x_{2}$, $x_{2}^{-1}, x_{3}$ and $x_{3}^{-1}$. This finds 68 new rules. When these rules are incorporated into the rewriting system we have a total of 7049 rules. By doing one further phase of overlap formation, using kb 10110 10, we now discover that we have 224196 rules. By using the rewrite_words command we find that the following newly-discovered relations hold: $x_{11}^{2}=\left[x_{10}, x_{1}, x_{2}\right]=\left[x_{10}, x_{1}, x_{3}\right]=\left[x_{10}, x_{3}\right]=1 ;\left[x_{10}, x_{1}, x_{1}\right]=x_{11}$; $\left[x_{9}, x_{3}\right]=x_{1} x_{10} x_{1}^{-1} x_{10}^{-1} ;\left[x_{7}, x_{2}\right]=x_{10}^{2} ;\left[x_{6}, x_{2}\right]=x_{3} x_{9} x_{3}^{-1} ;$ and $\left[x_{4}, x_{2}\right]=x_{8} x_{10}$. The total time for this computation on a Sun Sparcv9 machine with a $750 \mathrm{MHz}$ cpu running SunOS was $585.6 \mathrm{cpu}$ seconds.

This suffices to show that $T$ is nilpotent. The relation $x_{11}^{2}=1$ implies that we can assume $x_{11}=1$, using Section 2. Then the relations

$$
\left[x_{10}, x_{1}, x_{1}\right]=x_{11},\left[x_{10}, x_{1}, x_{2}\right]=\left[x_{10}, x_{1}, x_{3}\right]=1
$$

imply that $\left[x_{10}, x_{1}\right] \in \zeta(T)$, the centre of $T$. We showed above that $\left[x_{10}, x_{2}\right]=1$ so the fact that $\left[x_{10}, x_{1}\right] \in \zeta(T)$, together with the relation $\left[x_{10}, x_{3}\right]=1$, implies that $x_{10} \in \zeta_{2}(T)$, the second term of the ascending central series of $T$. Now $\left[x_{9}, x_{1}\right]=x_{11}=$ 1 , and we showed above that $\left[x_{9}, x_{2}\right]=1$, and so the relation $\left[x_{9}, x_{3}\right]=x_{1} x_{10} x_{1}^{-1} x_{10}^{-1}$ implies that $x_{9} \in \zeta_{2}(T)$. It follows easily from this that $T$ is nilpotent of class at most 5. Recall that the nilpotent quotient algorithm shows that any nilpotent quotient of $T$ is nilpotent of class at most 4 . So the fact that $T$ is nilpotent implies that its class is 4, even though our Knuth-Bendix calculation has not shown this directly.

However, although it is not necessary for our proof, we took relations revealed by this Knuth-Bendix run, and added them as defining relations to our input group. Here we did not assume that $x_{11}=1$, but rather we included the relations $\left[x_{11}, x_{1}\right]=$ $\left[x_{11}, x_{2}\right]=\left[x_{11}, x_{3}\right]=1$ which were also found. With these additional relations, and now using the wreath-product ordering, the Knuth-Bendix procedure quickly produced a confluent rewriting system for a nilpotent group of class 4 (in $181.8 \mathrm{cpu}$ seconds).

To enhance confidence in the Knuth-Bendix computations we have run equivalent calculations using the KBMAG package in MAGMA and obtained corresponding results. 


\section{$5 \quad$ Groups generated by three conjugate elements}

Let $E$ be a 4-Engel group with trivial Hirsch-Plotkin radical. Our aim is to prove that $E$ is trivial, or (equivalently) to prove that $E$ is locally nilpotent. The main step in our proof of Theorem 1 is to prove that if $a, x, y$ are three elements in $E$, then the subgroup $\left\langle a, a^{x}, a^{y}\right\rangle$ is nilpotent of class at most 5 . We establish this result in a series of steps. First we prove that $\left\langle a, a^{a^{a^{x}}}, a^{a^{y}}\right\rangle$ and $\left\langle a, a^{a^{a^{x}}},\left(a^{a^{a^{x}}}\right)^{a^{y}}\right\rangle$ are nilpotent. We use this result to prove that $\left\langle a, a^{a^{a^{x}}}, a^{y}\right\rangle$ is nilpotent. Next we show that $\left\langle a, a^{a^{x}}, a^{a^{y}}\right\rangle$ and $\left\langle a, a^{a^{x}},\left(a^{a^{x}}\right)^{a^{y}}\right\rangle$ are nilpotent. We make use of these results to prove that $\left\langle a, a^{a^{x}}, a^{y}\right\rangle$ is nilpotent, and then finally prove that $\left\langle a, a^{x}, a^{y}\right\rangle$ is nilpotent. Once we know that one of these groups is nilpotent we can use the nilpotent quotient algorithm to obtain bounds on the nilpotency class of the group. We repeatedly use the fact that if $a$ and $b$ are conjugate elements in a 4-Engel group then the subgroup generated by $a$ and $b$ is nilpotent of class at most 3. This follows from Traustason's result that 2-generator, 4Engel groups are nilpotent. We also make repeated use of the fact that $\langle a, x, y\rangle$ has no $\{2,3,5\}$-elements, which follows from our assumption that $E$ has trivial Hirsch-Plotkin radical. Finally, we need the following lemma.

Lemma 5. Let $a, y$ be elements in a 4-Engel group, and let $c=a^{y}, d=a^{y^{2}}, e=a^{y^{-1}}$. Then

(i) the normal closure of a in $\langle a, y\rangle$ is nilpotent of class at most 3 , and

(ii) $[c, a, c]^{8}=[c, a, a]^{-34}[d, a, a]^{6}[e, a, a]^{-30}$.

Proof. Traustason's theorem implies that the group $\langle a, y\rangle$ is nilpotent, and these results can be "read off" from a PCP for the free 4-Engel group on two generators.

We remarked above that once we have established that a subgroup $G \leq E$ is nilpotent, we use the nilpotent quotient algorithm to obtain a bound on the class of $G$. However the bounds obtained for the classes of the subgroups under consideration are never more than 5 , and it would be perfectly possible to obtain "hand" proofs of these bounds using commutator calculus or Lie ring methods.

\subsection{The subgroups $\left\langle a, a^{a^{a^{x}}}, a^{a^{y}}\right\rangle$ and $\left\langle a, a^{a^{a^{x}}},\left(a^{a^{a^{x}}}\right)^{a^{y}}\right\rangle$}

We let $b=a^{a^{a^{x}}}$ and let $c=a^{a^{y}}$. Since $\left\langle a, a^{x}\right\rangle$ is nilpotent of class at most 3 it follows that $[a, b]=1$, and since $\left\langle a, a^{y}\right\rangle$ is nilpotent of class at most 3 it follows that $[c, a, a]=[c, a, c]=1$. Since $b$ and $c$ are conjugate we have

$$
[c, b, b, b]=[c, b, b, c]=[c, b, c, c]=1 .
$$

So $\left\langle a, a^{a^{a^{x}}}, a^{a^{y}}\right\rangle$ is a homomorphic image of $T$, and is nilpotent of class at most 4 .

Similarly we see that $\left\langle a, a^{a^{a^{x}}},\left(a^{a^{a^{x}}}\right)^{a^{y}}\right\rangle$ is nilpotent of class at most 4 . We let $b=a^{a^{a^{x}}}$ and let $c=\left(a^{a^{a^{x}}}\right)^{a^{y}}$. Then $c=b^{a^{y}}=b^{b^{z}}$ for some $z$, since $a$ and $b$ are 
conjugate. So $[c, b, b]=[c, b, c]=1$. As above, $[a, b]=1$, and since $a$ and $c$ are conjugate we have $[c, a, a, a]=[c, a, a, c]=[c, a, c, c]=1$. So $\left\langle a, a^{a^{a^{x}}},\left(a^{a^{a^{x}}}\right)^{a^{y}}\right\rangle$ is a homomorphic image of $T$ and is nilpotent of class at most 4 .

\subsection{The subgroup $\left\langle a, a^{a^{a^{x}}}, a^{y}\right\rangle$}

We let $G=\langle a, b, c\rangle$ where $b=a^{a^{a^{x}}}$ and $c=a^{y}$. Since $\left\langle a, a^{x}\right\rangle$ is nilpotent of class at most 3 it follows that $[a, b]=1$. And since $a$ and $b$ are both conjugate to $c$ it follows that $\langle a, c\rangle$ and $\langle b, c\rangle$ are nilpotent of class at most 3. We use the results of Section 5.1 to establish some further identities.

Step 1: Show that $\langle[c, a, a],[c, a, c], b\rangle$ is nilpotent of class at most 2 .

First we show that $[c, a, a, b, b]=1$. From Lemma 5 we know that the normal closure of $a$ in the group $\langle a, y\rangle$ is nilpotent of class 3 (or less). So

$$
[c, a, a]=\left[a^{y}, a, a\right]=\left[a,\left[a, a^{y}\right]\right]=a^{-1} a^{a^{a^{y}}} .
$$

Since $a$ and $b$ commute, and since $a$ commutes with $a^{a^{a^{y}}}$ we obtain

$$
[c, a, a, b, b]=\left[a^{-1} a^{a^{a^{y}}}, a^{-1} b, a^{-1} b\right]=\left[a^{-1} a^{a^{a^{y}}},\left[a, a^{a^{x}}\right],\left[a, a^{a^{x}}\right]\right] \in \gamma_{5}\left\langle a, a^{a^{a^{y}}}, a^{a^{x}}\right\rangle .
$$

Now, from the results in Section 5.1 (with $x$ and $y$ interchanged), we know that $\left\langle a, a^{a^{a^{y}}}, a^{a^{x}}\right\rangle$ is nilpotent of class 4 or less. So

$$
[c, a, a, b, b]=1
$$

Next we show that $[c, b, b, a, a]=1$. Using the fact that $b=a^{a^{a^{x}}}$, we see that

$$
[c, b, b]=\left[c^{\prime}, a, a\right]^{a^{a^{x}}}
$$

where $c^{\prime}=a^{z}$ for some $z$. So

$$
[c, b, b, a, a]=\left[c^{\prime}, a, a, a^{\left(a^{a^{x}}\right)^{-1}}, a^{\left(a^{a^{x}}\right)^{-1}}\right]^{a^{a^{x}}} .
$$

Now, as above,

$$
\left[c^{\prime}, a, a\right]=a^{-1} a^{a^{a^{z}}}
$$

Also,

$$
a^{\left(a^{a^{x}}\right)^{-1}}=a\left[a,\left(a^{a^{x}}\right)^{-1}\right]=a\left[a^{a^{x}}, a\right] .
$$

So

$$
\left[c^{\prime}, a, a, a^{\left(a^{a^{x}}\right)^{-1}}, a^{\left(a^{a^{x}}\right)^{-1}}\right]=\left[a^{-1} a^{a^{a^{z}}}, a\left[a^{a^{x}}, a\right], a\left[a^{a^{x}}, a\right]\right]=\left[a^{a^{a^{z}}},\left[a^{a^{x}}, a\right],\left[a^{a^{x}}, a\right]\right]=1 .
$$

This establishes the identity

$$
[c, b, b, a, a]=1 .
$$


Next, we show that $\left[[c, a, c]^{8}, b, b\right]=1$. We have $c=a^{y}$, and we let $d=a^{y^{2}}$, $e=a^{y^{-1}}$. From Lemma 5 we have

$$
[c, a, c]^{8}=[c, a, a]^{-34}[d, a, a]^{6}[e, a, a]^{-30} .
$$

Also, Lemma 5 implies that the subgroup $\langle a, c, d, e\rangle$ is nilpotent of class at most 3 , so that if $u$ and $v$ are any two of $c, d, e$ then

$$
[u, a, a][v, a, a]=[v, a, a][u, a, a]
$$

and

$$
[u v, a, a]=[u, a, a][v, a, a]
$$

Also, if we let $f=a^{u v}$ then $\langle a, f\rangle$ is nilpotent of class at most 2, and $[u v, a, a]=[a, f]$. We let $H=\langle a, b, f\rangle$, and then $H$ is a homomorphic image of $T$. So $H$ is nilpotent of class at most 4 and

$$
[b,[u v, a, a],[u v, a, a]]=[b,[a, f],[a, f]]=1 .
$$

We let $K=\langle[u, a, a],[v, a, a], b\rangle$. Since $u$ and $v$ are conjugate to $a$ the relation $[c, a, a, b, b]=1$ implies that

$$
[[u, a, a], b, b]=[[v, a, a], b, b]=1,
$$

and the results of Section 5.1 imply that

$$
[[u, a, a], b,[u, a, a]]=[[v, a, a], b,[v, a, a]]=1 .
$$

So $K$ is also a homomorphic image of $T$, and $K$ is nilpotent. We also have the relation

$$
[b,[u, a, a][v, a, a],[u, a, a][v, a, a]]=1,
$$

and the nilpotent quotient algorithm implies that these relations give

$$
[[u, a, a], b,[v, a, a]]^{4}=1 .
$$

Since $\langle a, x, y\rangle$ has no elements of order 2 , this gives

$$
[[u, a, a], b,[v, a, a]]=1 .
$$

These relations imply that $K$ has class at most 2 , thus $\langle[c, a, a],[d, a, a],[e, a, a], b\rangle$ has class at most 2 . So we have

$$
\left[[c, a, a]^{-34}[d, a, a]^{6}[e, a, a]^{-30}, b, b\right]=1,
$$

and Lemma 5 gives $\left[[c, a, c]^{8}, b, b\right]=1$. Similarly

$$
\left[[c, a, c]^{8}, b,[c, a, c]^{8}\right]=1 .
$$

These relations imply that in the (nilpotent) 2-generator, 4-Engel group $M=\langle[c, a, c], b\rangle$, $\gamma_{3}(M) / \gamma_{4}(M)$ is a 2-group. It follows that $\gamma_{3}(M)$ is a 2-group, and hence that $\gamma_{3}(M)=\{1\}$. So $\langle[c, a, c], b\rangle$ is nilpotent of class at most 2 .

Similarly, $\langle[c, b, c], a\rangle$ is nilpotent of class at most 2 .

So $a, b, c$ satisfy the following properties: 
(i) $\langle a, b\rangle$ is abelian,

(ii) $\langle a, c\rangle$ and $\langle b, c\rangle$ have class at most 3 since $a, b, c$ are conjugate,

(iii) $\left\langle a, b, a^{c}\right\rangle$ and $\left\langle a, b, b^{c}\right\rangle$ have class at most 4 by the results of Section 5.1,

(iv) $\langle[c, a, a], b\rangle,\langle[c, b, b], a\rangle,\langle[c, a, c], b\rangle$ and $\langle[c, b, c], a\rangle$ are nilpotent of class at most 2 .

So in the group $L=\langle[c, a, a],[c, a, c], b\rangle$, the subgroup $\langle[c, a, a],[c, a, c]\rangle$ is abelian and the subgroups $\langle[c, a, a], b\rangle,\langle[c, a, c], b\rangle$ both have class at most 2 . It follows that $L$ is nilpotent of class at most 4 . The argument above also gives $\left[[c, a, a], b,[c, a, c]^{8}\right]=1$, and (given that $L$ is nilpotent) it follows from this that $[[c, a, a], b,[c, a, c]]^{32}=1$. But $\langle a, x, y\rangle$ has no 2-elements, and so $[[c, a, a], b,[c, a, c]]=1$, which implies that $L$ is nilpotent of class at most 2 .

This completes Step 1. We similarly see that $\langle[c, b, b],[c, b, c], a\rangle$ is nilpotent of class at most 2 .

Note that these properties are symmetric in $a$ and $b$. We show that these properties imply that $G$ is nilpotent. We assume throughout that the Hirsch-Plotkin radical of $G$ is trivial, so that $G$ has trivial centre and has no elements of order 2,3 or 5 .

The main step in the proof that $G$ is nilpotent is in showing that if the HirschPlotkin radical of $G$ is trivial then $[c, a, a]=1$. By symmetry we also have $[c, b, b]=1$. We then go on to show that $[c, a, c]=[c, b, c]=1$. So if the Hirsch-Plotkin radical of $G$ is trivial then $G$ is a homomorphic image of $T$, and $G$ is nilpotent.

Step 2: Show that $[c, a, a]=1$.

First we note that if we let $v=[c, a, a], u=c, w=b$ then $\langle u, v, w\rangle$ satisfies the relations of $T$, and so $\langle[c, a, a], c, b\rangle$ has class at most 4 . By symmetry $\langle[c, b, b], c, a\rangle$ has class at most 4 . Now consider the element $[[c, a, a],[c, b, b]]$. It lies in $\gamma_{4}\langle[c, a, a], c, b\rangle$ and so it is centralized by $b$ and $c$. It also lies in $\gamma_{4}\langle[c, b, b], c, a\rangle$ and so it is centralized by $a$ and $c$. So $[[c, a, a],[c, b, b]] \in \zeta(G)$. Under the assumption that $\zeta(G)=\{1\}$ we have

$$
[[c, a, a],[c, b, b]]=1 .
$$

Next we show that $\langle[c, b, c], c, a\rangle$ is nilpotent of class at most 4 . We let $v=[c, b, c]$, $u=c, w=a$. Then $[u, v]=1$ and $[w, v, v]=[w, v, w]=1$ since $\langle[c, b, b],[c, b, c], a\rangle$ has class at most 2 . So $\langle[c, b, c], c, a\rangle$ satisfies the relations of $T$, and is nilpotent of class at most 4 . By symmetry $\langle[c, a, c], c, b\rangle$ is nilpotent of class at most 4 .

Now consider $[c, a, a, b, c, c] \in \gamma_{4}\langle[c, a, a], c, b\rangle$. Since $\langle[c, a, a], c, b\rangle$ has class at most 4 , this element is centralized by $b$ and $c$. Also since $[c, a, a, c]=1$ we have

$$
[c, a, a, b, c, c]=[[c, a, a],[b, c, c]]=\left[[c, a, a],[c, b, c]^{-1}\right] \in \gamma_{4}\langle[c, b, c], c, a\rangle,
$$

and so $[c, a, a, b, c, c]$ is centralized by $a$ and $c$. It follows that $[c, a, a, b, c, c] \in \zeta(G)=$ $\{1\}$. 
Next consider $[c, a, a, b, c, a]$. We see that the subgroup $\langle[c, a, a, b], a, c\rangle$ satisfies the relations of $T$, and is nilpotent of class at most 4 . So

$$
[c, a, a, b, c, a, a]=[[c, a, a], b,[c, a, a]]=1 .
$$

Also, the subgroup $\langle[c, a, c], c, b\rangle$ has class at most 4 , and so the relation

$$
[[c, a, a],[c, b, b]]=1
$$

gives

$$
[c, a, a, c, b, b][c, a, a, b, c, b]^{-2}[c, a, a, b, b, c]=1 .
$$

Since $[c, a, a, c]=[c, a, a, b, b]=1$ we have $[c, a, a, b, c, b]=1$. So $b$ commutes with $[c, a, a, b, c]$, and since it also commutes with $a$ we have $[c, a, a, b, c, a, b]=1$.

Finally,

$$
[[c, a, a], b,[c, a, c]] \in \gamma_{3}\langle[c, a, a],[c, a, c], b\rangle=\{1\}
$$

SO

$$
\begin{aligned}
1 & =[c, a, a, b,[c, a, c]] \\
& =[c, a, a, b, a, c, c]^{-1}[c, a, a, b, c, a, c]^{2}[c, a, a, b, c, c, a]^{-1} \\
& =[c, a, a, b, c, a, c]^{2}
\end{aligned}
$$

We are assuming that $G$ has no elements of order 2 , thus $[c, a, a, b, c, a, c]=1$.

So $[c, a, a, b, c, a, z]=1$ for $z=a, b, c$, and $[c, a, a, b, c, a] \in \zeta(G)=\{1\}$.

We showed above that $[c, a, a, b, c, c]=1$, so consider $[c, a, a, b, c, b]$. Since $\langle[c, a, a], c, b\rangle$ has class at most 4 , and $[c, a, a, c]=[c, a, a, b, b]=1,[[c, a, a],[c, b, b]]=[c, a, a, b, c, b]^{-2}$.

Now we showed above that $[[c, a, a],[c, b, b]]=1$, and we are assuming that $G$ has no elements of order 2. So $[c, a, a, b, c, b]=1$. It follows that $[c, a, a, b, c, z]=1$ for $z=a, b, c$, and that $[c, a, a, b, c] \in \zeta(G)=\{1\}$. But $[c, a, a, b, a]=[c, a, a, b, b]=1$, and so $[c, a, a, b]=1$. It follows that $[c, a, a]=1$.

This completes Step 2. By symmetry $[c, b, b]=1$.

Step 3: Show that $[c, a, c]=1$.

We have $[c, a, c, a]=[c, a, c, c]=[c, a, c, b, b]=[c, a, c, b, a]=1$. So consider $[c, a, c, b, c]$. We know that $\langle[c, a, c], c, b\rangle$ is nilpotent of class at most 4 , and so (since $[c, b, b]=1)$

$$
1=[[c, a, c],[c, b, b]]=[c, a, c, b, c, b]^{-2} .
$$

So $[c, a, c, b, c, b]=1$. Also

$$
[c, a, c, b, c, c]=\left[[c, a, c],[c, b, c]^{-1}\right]
$$

is centralized by $b, c$ since $\langle[c, a, c], c, b\rangle$ is nilpotent of class at most 4 . And it is centralized by $a, c$ since $\langle[c, b, c], c, a\rangle$ is nilpotent of class at most 4 . So $[c, a, c, b, c, c] \in$ $\zeta(G)=\{1\}$. 
Next, using the fact that $\langle[c, a, c], c, b\rangle$ is nilpotent of class at most 4 and the fact that $[c, a, c, b, c, c]=1$, we see that $\langle[c, a, c, b], a, c\rangle$ is nilpotent of class at most 4 . So

$$
[c, a, c, b, c, a, a]=[c, a, c, b,[c, a, a]]=1 .
$$

And $[c, a, c, b, c, a, b]=1$ since $[c, a, c, b, c, b]=1$. Finally,

$$
[[c, a, c], b,[c, a, c]]=1
$$

and so, since $\langle[c, a, c, b], a, c\rangle$ is nilpotent of class at most 4 ,

$$
[c, a, c, b, c, a, c]^{-2}=1 .
$$

So $[c, a, c, b, c, a]=1$. This, together with the relation $[c, a, c, b, c, b]=[c, a, c, b, c, c]$ proved above, implies that $[c, a, c, b, c]=1$. This in turn proves that $[c, a, c, b]=1$, and finally that $[c, a, c]=1$.

This completes Step 3. By symmetry, $[c, b, c]=1$.

The relations $[a, b]=[c, a, a]=[c, b, b]=[c, a, c]=[c, b, c]=1$ imply that $G$ is a homomorphic image of $T$. So $G$ is nilpotent, as claimed.

Now that we know that $G$ is nilpotent we can use the nilpotent quotient algorithm to obtain information about it, using the following properties which were established without the assumption that the Hirsch-Plotkin radical of $G$ is trivial:

(i) $\langle a, b\rangle$ is abelian,

(ii) $\langle a, c\rangle$ and $\langle b, c\rangle$ are nilpotent of class at most 3,

(iii) $[c, a, a, b, b]=[c, b, b, a, a]=[c, a, c, b, b]=[c, b, c, a, a]=1$.

Given that $G$ has no 2-elements, these properties imply that $G$ has class at most 4. They also imply that $\gamma_{4}(G)$ is generated by $[c, b, a, c],[c, a, a, b],[c, b, b, a],[c, a, c, b]$, $[c, b, c, a]$, and that

$$
[c, b, a, c]^{2}=[c, a, c, b][c, b, c, a] .
$$

Step 4: Show that these properties imply that $\gamma_{4}\left\langle a, a^{a^{a^{x}}}, a^{y}\right\rangle \leq \zeta\left\langle a, a^{x}, a^{y}\right\rangle$.

We let $G=\langle a, b, c\rangle$ where $b=a^{a^{a^{x}}}$ and $c=a^{y}$, and we make use of the properties of $G$ established above.

First we show that $[c, a, a, b]$ and $[c, b, b, a]$ lie in the centre of $\left\langle a, a^{x}, a^{y}\right\rangle$.

We have $[c, a, a]=a^{-1} a^{a^{a^{y}}}$ and so $[c, a, a, b]=\left[a^{a^{a^{y}}}, a^{a^{a^{x}}}\right]$. We know that this element lies in the centre of $G$, so that it is centralized by $a$ and $a^{y}$. By symmetry it is also centralized by $a^{x}$. So $[c, a, a, b] \in \zeta\left\langle a, a^{x}, a^{y}\right\rangle$.

Now consider $[c, b, b, a]$. We have

$$
[c, b, b, a]=\left[c^{z^{-1}}, a, a, a^{z^{-1}}\right]^{z}
$$

where $z=a^{a^{x}}$. It is straightforward to show that $a^{z^{-1}}=a^{2} b^{-1}$ and so

$$
[c, b, b, a]=\left[c^{z^{-1}}, a, a, b^{-1}\right]^{z}=\left[c^{z^{-1}}, a, a, b\right]^{-z} .
$$


We showed above that $[c, a, a, b]$ is centralized by $a$ and $a^{x}$, and substituting $c^{z^{-1}}$ for $c$ we see that this implies that $\left[c^{z^{-1}}, a, a, b\right]$ is centralized by $a$ and $a^{x}$. So $[c, b, b, a]=$ $\left[c^{z^{-1}}, a, a, b\right]^{-1}$, and $[c, b, b, a]$ is also centralized by $a$ and $a^{x}$. Since $[c, b, b, a] \in \zeta(G)$ it is centralized by $c=a^{y}$. So $[c, b, b, a] \in \zeta\left\langle a, a^{x}, a^{y}\right\rangle$.

Next, we show that $[c, a, c, b]$ and $[c, b, c, a]$ lie in the centre of $\left\langle a, a^{x}, a^{y}\right\rangle$.

We let $c=a^{y}$, and we let $d=a^{y^{2}}, e=a^{y^{-1}}$. Then by Lemma 5 , together with the facts proved above that $\langle[c, a, c], b\rangle$ and $\langle[c, a, a],[d, a, a],[e, a, a], b\rangle$ are nilpotent of class at most 2 , we have

$$
[c, a, c, b]^{8}=\left[[c, a, c]^{8}, b\right]=[c, a, a, b]^{-34}[d, a, a, b]^{6}[e, a, a, b]^{-30} .
$$

We proved above that $[c, a, a, b]$ is centralized by $a^{x}$, and this also implies that $[d, a, a, b]$ and $[e, a, a, b]$ are centralized by $a^{x}$. So $[c, a, c, b]^{8}$ is centralized by $a^{x}$. In the nilpotent 2-generator group $M=\left\langle[c, a, c, b], a^{x}\right\rangle$ this implies that $\gamma_{2}(M) / \gamma_{3}(M)$ is a 2-group, and hence that $\gamma_{2}(M)$ is a 2-group. So $M$ is abelian, and $[c, a, c, b]$ is centralized by $a^{x}$. Since $G$ has class at most 4 , we know that $[c, a, c, b]$ is centralized by $a$ and $c=a^{y}$, and so $[c, a, c, b] \in \zeta\left\langle a, a^{x}, a^{y}\right\rangle$.

The proof that $[c, b, c, a] \in \zeta\left\langle a, a^{x}, a^{y}\right\rangle$ is similar.

Finally, note that the fact that

$$
[c, b, a, c]^{2}=[c, a, c, b][c, b, c, a]
$$

implies that $[c, b, a, c]^{2} \in \zeta\left\langle a, a^{x}, a^{y}\right\rangle$, and hence that $[c, b, a, c] \in \zeta\left\langle a, a^{x}, a^{y}\right\rangle$.

Summarizing, $[c, b, a, c],[c, a, a, b],[c, b, b, a],[c, a, c, b],[c, b, c, a] \in \zeta\left\langle a, a^{x}, a^{y}\right\rangle$, which implies that $\gamma_{4}\langle a, b, c\rangle \leq \zeta\left\langle a, a^{x}, a^{y}\right\rangle$.

\subsection{The subgroups $\left\langle a, a^{a^{x}}, a^{a^{y}}\right\rangle$ and $\left\langle a, a^{a^{x}},\left(a^{a^{x}}\right)^{a^{y}}\right\rangle$}

We let $b=a^{a^{x}}, c=a^{a^{y}}$, and consider the group $G=\langle a, b, c\rangle$. We show that $G$ is nilpotent.

Since $\left\langle a, a^{x}\right\rangle,\left\langle a, a^{y}\right\rangle$ and $\langle b, c\rangle$ are all nilpotent of class at most 3 we have

$$
\begin{gathered}
{[b, a, a]=[b, a, b]=[c, a, a]=[c, a, c]=1,} \\
{[b, c, c, c]=[b, c, c, b]=[b, c, b, b]=1 .}
\end{gathered}
$$

Also

$$
[c, a, b, a],[c, a, b, b],[c, a, b,[c, a]] \in \gamma_{4}\left\langle a, a^{a^{a^{y}}}, a^{x}\right\rangle
$$

and

$$
[b, a, c, a],[b, a, c, c],[b, a, c,[b, a]] \in \gamma_{4}\left\langle a, a^{a^{a^{x}}}, a^{y}\right\rangle,
$$

so that the results in Section 5.2 imply that

$$
[c, a, b, a],[c, a, b, b],[b, a, c, a],[b, a, c, c],[b, a, c,[b, a]],[c, a, b,[c, a]]
$$

lie in the centre of $G$. 
We assume for the moment that the Hirsch-Plotkin radical of $G$ is trivial, so that $[c, a, b, a]=[c, a, b, b]=[b, a, c, a]=[b, a, c, c]=[b, a, c,[b, a]]=[c, a, b,[c, a]]=1$.

Now consider $[b, a, c, b, a]$. Using the Jacobi-Witt identity we see that

$$
[[b, a, c], b, a]^{b^{-1}}\left[b^{-1}, a^{-1},[b, a, c]\right]^{a}\left[a,[b, a, c]^{-1}, b^{-1}\right]^{[b, a, c]}=1 .
$$

Since $\left[b^{-1}, a^{-1}\right]=[b, a]$ we see that $[b, a, c, b, a]=1$.

We also have $[b, a, c, b, b],[b, a, c, b, c] \in \gamma_{4}\left\langle b, b^{a}, c\right\rangle$. Now $b=a^{a^{x}}$ and so $a^{x}=$ $b^{z}$ for some $z$ and $a=b^{\left(b^{z}\right)^{-1}}$. It follows that $b^{a}=b^{2} d^{-1}$ where $d=b^{b^{b^{z}}}$ and so $\left\langle b, b^{a}, c\right\rangle=\left\langle b, b^{b^{b^{z}}}, c\right\rangle$. So the results in Section 5.2 imply that $[b, a, c, b, b],[b, a, c, b, c]$ are centralized by $b, b^{z}$ and $c$. So $[b, a, c, b, b],[b, a, c, b, c] \in \zeta(G)$. Since the HirschPlotkin radical of $G$ is trivial this implies that $[b, a, c, b, b]=[b, a, c, b, c]=1$. But we showed above that $[b, a, c, b, a]=1$, and so $[b, a, c, b] \in \zeta(G)$. But this implies that $[b, a, c, b]=1$, which together with $[b, a, c, a]=[b, a, c, c]=1$ implies that $[b, a, c]=1$ and hence that $[b, a]=1$. So $G$ satisfies the relations of $T$ and $G$ is nilpotent.

Now we lift the assumption that the Hirsch-Plotkin radical of $G$ is trivial. We have the relations (3) and (4), and the elements (5) lie in the centre of $G$. These relations imply that $G$ has class at most 4 .

Similarly, $H=\left\langle a, a^{a^{x}},\left(a^{a^{x}}\right)^{a^{y}}\right\rangle$ has class at most 4 . To see this we note that if we let $b=a^{a^{x}}, c^{\prime}=\left(a^{a^{x}}\right)^{a^{y}}$, then we can write $a^{x}=b^{z}, a^{y}=b^{t}$ for some $z, t$. So $a=b^{\left(b^{z}\right)^{-1}}$ and $c^{\prime}=b^{b^{t}}$. The elements $\left[c^{\prime}, b, a, b\right],\left[c^{\prime}, b, a, a\right],\left[c^{\prime}, b, a,\left[c^{\prime}, b\right]\right]$ lie in $\gamma_{4}\left\langle b, b^{b^{b^{t}}}, b^{z}\right\rangle$, and the elements $\left[a, b, c^{\prime}, b\right],\left[a, b, c^{\prime}, c^{\prime}\right],\left[a, b, c^{\prime},[a, b]\right]$ lie in $\gamma_{4}\left\langle b, b^{b^{b^{z}}}, b^{t}\right\rangle$, so the proof that $H$ is nilpotent goes through in the same way as the proof that $G$ is nilpotent.

\subsection{The subgroup $\left\langle a, a^{a^{x}}, a^{y}\right\rangle$}

We let $b=a^{a^{x}}$ and let $c=a^{y}$, and consider the group $G=\langle a, b, c\rangle$. We prove that this group is nilpotent. Clearly $\langle a, b\rangle$ is nilpotent of class at most 2, and $\langle a, c\rangle$ and $\langle b, c\rangle$ are nilpotent of class at most 3. The results of Section 5.3 imply that $\left\langle a, b, a^{c}\right\rangle$ and $\left\langle a, b, b^{c}\right\rangle$ are nilpotent of class at most 4 .

We consider the subgroup $\langle[c, a, a], a, b\rangle$. We have $[c, a, a]=a^{-1} a^{a^{c}}$ and so

$$
\langle[c, a, a], a, b\rangle=\left\langle a, a^{a^{c}}, b\right\rangle=\left\langle a, a^{a^{a^{y}}},\left[a, a^{x}\right]\right\rangle .
$$

So

$$
\gamma_{3}\langle[c, a, a], a, b\rangle \leq \gamma_{4}\left\langle a, a^{a^{a^{y}}}, a^{x}\right\rangle
$$

and by the results of Section 5.2

$$
\gamma_{3}\langle[c, a, a], a, b\rangle \leq \zeta(G)
$$

Similarly, the following subgroups all lie in the centre of $G$ :

$$
\gamma_{3}\langle[c, b, b], a, b\rangle, \gamma_{4}\langle[a, b], a, c\rangle, \gamma_{4}\langle[a, b], b, c\rangle, \gamma_{4}\langle[c, a, c], b, c\rangle, \gamma_{4}\langle[c, b, c], a, c\rangle .
$$


We show that these properties imply that $G$ is nilpotent. In proving this, we can assume that the Hirsch-Plotkin radical of $G$ is trivial, so that the centre of $G$ is trivial. So we can assume that $G$ satisfies the following properties:

(i) $\langle a, b\rangle$ is nilpotent of class at most 2 ,

(ii) $\langle a, c\rangle$ and $\langle b, c\rangle$ are nilpotent of class at most 3,

(iii) $\left\langle a, b, a^{c}\right\rangle$ and $\left\langle a, b, b^{c}\right\rangle$ are nilpotent of class at most 4 ,

(iv) $\langle[c, a, a], a, b\rangle$ and $\langle[c, b, b], a, b\rangle$ are nilpotent of class at most 2 ,

(v) $\langle[a, b], a, c\rangle$ and $\langle[a, b], b, c\rangle$ are nilpotent of class at most 3 ,

(vi) $\langle[c, a, c], b, c\rangle$ and $\langle[c, b, c], a, c\rangle$ are nilpotent of class at most 3.

Note that these properties are symmetrical in $a$ and $b$.

Step 1: Prove that $\langle[c, a, a], c, b\rangle$ and $\langle a,[c, a, c], b\rangle$ are nilpotent of class at most 3 .

If we let $v=[c, a, a], u=c, w=b$ then

$$
[u, v]=[w, v, v]=[w, v, w]=[w, u, u, u]=[w, u, u, w]=[w, u, w, w]=1
$$

and so $\langle u, v, w\rangle$ satisfies the relations of $T$ and is nilpotent of class at most 4 . So

$$
[[c, a, a],[c, b, b], b]=[[c, a, a],[c, b, b], c]=1 .
$$

By symmetry between $a$ and $b$ we also have

$$
[[c, a, a],[c, b, b], a]=1
$$

and so $[[c, a, a],[c, b, b]]$ is central, and therefore trivial. Since $\langle[c, b, c], a, c\rangle$ is nilpotent of class at most 3 we also have $[[c, a, a],[c, b, c]]=1$. We also have $[c, a, a, c]=$ $[c, a, a, b, b]=1$, and so using the fact that $\langle[c, a, a], c, b\rangle$ has class at most 4 we obtain

$$
\begin{aligned}
1 & =[c, a, a,[c, b, b]] \\
& =[c, a, a, c, b, b][c, a, a, b, c, b]^{-2}[c, a, a, b, b, c] \\
& =[c, a, a, b, c, b]^{-2}
\end{aligned}
$$

and hence $[c, a, a, b, c, b]=1$. Using $[[c, a, a],[c, b, c]]=1$ we similarly obtain

$$
[c, a, a, b, c, c]=1 \text {. }
$$

We also have

$$
[[c, a, a], b,[c, a, a]] \in \gamma_{5}\left\langle a, b, a^{c}\right\rangle=\{1\}
$$

and so, in fact, $\langle[c, a, a], c, b\rangle$ has class at most 3 .

Similarly, $\langle a,[c, a, c], b\rangle$ is a homomorphic image of $T$ (mapping $v$ to the first generator, $u$ to the second, and $w$ to third), and so $\langle a,[c, a, c], b\rangle$ is nilpotent of class at 
most 4 . We also have $[[a, b],[c, a, c]]=1$ since $\langle[a, b], a, c\rangle$ is nilpotent of class at most 3 , and this implies that $\langle a,[c, a, c], b\rangle$ has class at most 3 .

Step 2: Show that $[c, a, a]=1$.

Since $\langle a,[c, a, c], b\rangle$ has class at most 3 , we have

$$
[c, a, c, b, b, a]=[c, a, c, b, b, b]=1 .
$$

We also have $[c, a, c, b, b, c]=1$ since $\langle[c, a, c], c, b\rangle$ is nilpotent of class at most 3 , and so $[c, a, c, b, b]$ is central, and hence trivial.

Since $\langle[c, a, c], c, b\rangle$ is nilpotent of class at most 3 , it follows that $[[c, a, c], b,[c, a, c]]$ is centralized by $b$ and $c$. We also have

$$
\begin{aligned}
{[[c, a, c], b,[c, a, c]]^{a} } & =\left[[c, a, c], b^{a},[c, a, c]\right] \\
& =[[c, a, c], b[b, a],[c, a, c]] \\
& =[[c, a, c], b,[c, a, c]]
\end{aligned}
$$

since $[b, a]$ commutes with $[c, a, c]$ and $b$. So $[[c, a, c], b,[c, a, c]]$ is central and therefore trivial. It follows that $\langle[c, a, c], b\rangle$ has class at most 2. Furthermore the group $\langle[c, a, a],[c, a, c], b\rangle$ is a homomorphic image of $T$ since $\langle[c, a, a],[c, a, c]\rangle$ is abelian and $\langle[c, a, a], b\rangle$ and $\langle[c, a, c], b\rangle$ have class at most 2. So $\langle[c, a, a],[c, a, c], b\rangle$ is nilpotent, and the relations imply that it has class at most 3 (given that $G$ has no elements of order 2). So

$$
[[c, a, a], b,[c, a, c], b]=1 .
$$

Now consider $\langle[c, a, a, b], a, c\rangle$. Using the fact that $\langle[c, a, a], a, b\rangle$ has class at most 2 , and the fact that $\langle[c, a, a], c, b\rangle$ has class at most 3 , we see that this subgroup is a homomorphic image of $T$ and so is nilpotent of class at most 4 . So

$$
\begin{aligned}
{[c, a, a, b, c, a] } & =[c, a, a, b, c]^{-1}[c, a, a, b, c]^{a} \\
& =[c, a, a, b, c]^{-1}\left[c, a, a, b, c^{a}\right] \\
& =[c, a, a, b, c]^{-1}[c, a, a, b, c[c, a]] \\
& =[c, a, a, b,[c, a]][c, a, a, b, c,[c, a]] .
\end{aligned}
$$

We have $[c, a, a, b,[c, a], b] \in \gamma_{5}\left\langle a, b, a^{c}\right\rangle=\{1\}$, and so

$$
[c, a, a, b, c, a, b]=[c, a, a, b, c,[c, a], b]=[c, a, a, b, c, a, c, b]^{-1}
$$

using the fact that $[c, a, a, b, c, c]=1$. We also have

$$
1=[[c, a, a], b,[c, a, c], b]=\left[[c, a, a, b, c, a, c]^{2}, b\right] .
$$

Since $\langle[c, a, a, b], a, c\rangle$ has class at most 4 we have

$$
[c, a, a, b, c, a, c, a]=[c, a, a, b, c, a, c, c]=1,
$$

and so $[c, a, a, b, c, a, c]^{2}$ is central, which implies that $[c, a, a, b, c, a, c]=1$. So the relation (6) above gives $[c, a, a, b, c, a, b]=1$. Also

$$
[c, a, a, b, c, a, a]=[[c, a, a], b,[c, a, a]]=1 .
$$


So $[c, a, a, b, c, a]$ is central and therefore trivial. We showed above that $\langle[c, a, a], b, c\rangle$ has class at most 3 , and so this implies that $[c, a, a, b, c]$ is central and therefore trivial. This in turn implies that $[c, a, a, b]=1$, which (since $[c, a, a, a]=[c, a, a, c]=1$ ) implies $[c, a, a]=1$.

This completes Step 2. By symmetry we have $[c, b, b]=1$.

Step 3: Show that $[a, b]=1$.

We know that $\langle[a, b], a, c\rangle$ has class at most 3 , and so

$$
[a, b, c, c, a]=[a, b, c, c, c]=1 .
$$

We also know that $\langle[a, b], b, c\rangle$ has class at most 3 and so $[a, b, c, c, b]=1$. It follows that $[a, b, c, c]=1$. Similarly $[[a, b], c,[a, b]]=1$.

Now let $x, y, z$ be any elements of $G$. Then

$$
x y z=y z x[x, y z]=z x y[y, z x][x, y z]=x y z[z, x y][y, z x][x, y z] .
$$

So

$$
[z, x y][y, z x][x, y z]=1 .
$$

Substituting $[a, b, c]$ for $x$, and substituting $a, b$ for $y, z$ respectively, we obtain

$$
[b, a][a, b, c, b]^{-a}[a, b, c, a]^{-1}[a, b][[a, b],[a, b, c]][a, b, c, b][a, b, c, a]^{b}=1 .
$$

Now, as we showed above, $[[a, b],[a, b, c]]=1$, and since $[a, b]$ commutes with $a$ and $b$ we see that $[a, b]$ commutes with $[a, b, c, a]$ and $[a, b, c, b]$. So

$$
[a, b, c, b]^{-a}[a, b, c, a]^{-1}[a, b, c, b][a, b, c, a]^{b}=1 .
$$

Now $[a, b, c, b]^{-a}=[a, b, c, b, a]^{-1}[a, b, c, b]^{-1}$ and $[a, b, c, a]^{b}=[a, b, c, a][a, b, c, a, b]$. So we obtain

$$
[a, b, c, b, a]^{-1}[[a, b, c, b],[a, b, c, a]][a, b, c, a, b]=1,
$$

which is equivalent to

$$
[[a, b, c, b],[a, b, c, a]]=[a, b, c, b, a][a, b, c, a, b]^{-1} .
$$

Now $[[[a, b, c, b],[a, b, c, a]], c]=1$ since $\langle[a, b], a, c\rangle$ and $\langle[a, b], b, c\rangle$ have class at most 3 , and so $\left[[a, b, c, b, a][a, b, c, a, b]^{-1}, c\right]=1$. Also, since $\langle[a, b], b, c\rangle$ has class at most 3 ,

$$
[a, b, c, b]=[a, b,[c, b]]=\left[a, b, b^{-c} b\right]
$$

and so

$$
[a, b, c, b, a, a],[a, b, c, b, a, b] \in \gamma_{5}\left\langle a, b, b^{c}\right\rangle=\{1\} .
$$

So $[a, b, c, b, a]$ is centralized by $a$ and $b$. Similarly $[a, b, c, a, b]$ is centralized by $a$ and b. Hence

$$
\left[[a, b, c, b, a][a, b, c, a, b]^{-1}, x\right]=1
$$


for $x=a, b, c$, which implies that

$$
[a, b, c, b, a]=[a, b, c, a, b]
$$

Now consider the subgroup $\langle[a, b, c, a], c, b\rangle$. Let $v=[a, b, c, a], u=c, w=b$. Then $[u, v]=1$, since $\langle[a, b], a, c\rangle$ has class at most 3 . Also, using the fact that $\langle[a, b], a, c\rangle$ has class at most 3 again, we have

$$
[a, b, c, a]=[a, b,[c, a]]=\left[a, b, a^{-c} a\right]
$$

so that $[w, v, v]=[w, v, w]=1$. Finally, $\langle u, w\rangle$ has class at most 3. So $\langle[a, b, c, a], c, b\rangle$ is a homomorphic image of $T$, and is nilpotent of class at most 4 . So

$$
[a, b, c, a, b, c, c, b]=[a, b, c, a, b, c, c, c]=1 .
$$

By symmetry between $a$ and $b,\langle[a, b, c, b], c, a\rangle$ also has class at most 4 , which implies that

$$
[a, b, c, b, a, c, c, a]=1 .
$$

But then the fact that $[a, b, c, b, a]=[a, b, c, a, b]$ implies that $[a, b, c, a, b, c, c, a]=1$. So $[a, b, c, a, b, c, c]$ is central, which gives

$$
[a, b, c, a, b, c, c]=[a, b, c, b, a, c, c]=1 \text {. }
$$

We know that $[a, b, c, a, c]=1$, and that $[a, b, c, a, b, b] \in \gamma_{5}\left\langle a, b, a^{c}\right\rangle=\{1\}$, so

$$
1=[a, b, c, a,[c, b, b]]=[a, b, c, a, b, c, b]^{-2},
$$

and hence $[a, b, c, a, b, c, b]=1$. By symmetry between $a$ and $b$, we also have

$$
[a, b, c, a, b, c, a]=[a, b, c, b, a, c, a]=1 .
$$

Hence $[a, b, c, a, b, c]=1$, and since $[a, b, c, a, b, a]=[a, b, c, a, b, b]=1$, it follows that $[a, b, c, a, b]=1$. Since $[a, b, c, a, a]=[a, b, c, a, c]=1$, this implies that $[a, b, c, a]=1$. By symmetry between $a$ and $b,[a, b, c, b]=1$ also. We showed above that $[a, b, c, c]=1$, and so $[a, b, c]=1$. Since $[a, b, a]=[a, b, b]=1$ we finally obtain $[a, b]=1$, as required. This completes Step 3.

Step 4: Prove that $[c, a, c]=1$.

Since $\langle[c, a, c], b, c\rangle$ is nilpotent of class at most 3 , we obtain

$$
[c, a, c, b, b, b]=[c, a, c, b, b, c]=1,
$$

and since $a$ commutes with $b$ and $[c, a, c]$ we have

$$
[c, a, c, b, b, a]=1 \text {. }
$$

So $[c, a, c, b, b]=1$. 
We also have $[c, a, c, b, c, b]=[c, a, c, b, c, c]=1$, and so we consider $[c, a, c, b, c, a]$. The group $\langle[c, a, c, b], a, c\rangle$ is a homomorphic image of $T$, and so is nilpotent of class at most 4 . So

$$
[c, a, c, b, c, a, a]=[c, a, c, b,[c, a, a]]=1 .
$$

Also

$$
[c, a, c, b, c, a, b]=1
$$

since $[a, b]=[c, a, c, b, c, b]=1$, and we showed above that $[[c, a, c], b,[c, a, c]]=1$ and SO

$$
[c, a, c, b, c, a, c]^{-2}=1 .
$$

This implies that $[c, a, c, b, c, a]=1$, and hence that $[c, a, c, b, c]=1$. We showed above that $[c, a, c, b, b]=1$, and since $[a, b]=[c, a, c, a]=1$ we have $[c, a, c, b, a]=1$. It follows that $[c, a, c, b]=1$ and finally that $[c, a, c]=1$.

This completes Step 4. So $[a, b]=[c, a, a]=[c, b, b]=[c, a, c]=1$, and by symmetry in $a$ and $b$ we also have $[c, b, c]=1$. So $G$ is a homomorphic image of $T$ and $G$ is nilpotent.

Note that the conclusion that $G$ is a homomorphic image of $T$ is based on the assumption that the Hirsch-Plotkin radical of $G$ is trivial. We now lift this assumption, and use the nilpotent quotient algorithm to compute $G$ using the fact that it is a nilpotent 4-Engel group satisfying the following properties:

(i) $\langle a, b\rangle$ has class 2 ,

(ii) $\langle a, c\rangle,\langle b, c\rangle,\left\langle a, b^{c}\right\rangle,\left\langle b, c^{a}\right\rangle,\left\langle c, a^{b}\right\rangle$ have class 3 .

The computation shows that $G$ has class at most 5 , and that $[c, a, a,[b, a]]=$ $[c, b, b,[b, a]]=1$. These relations, together with Lemma 5 imply that

$$
\left[[c, a, c]^{8},[b, a]\right]=\left[[c, b, c]^{8},[b, a]\right]=1,
$$

and hence that

$$
[[c, a, c],[b, a]]=[[c, b, c],[b, a]]=1,
$$

Adding these two relations we see that $\gamma_{5}(G)$ is a $\{2,3,5\}$-group, so that $G$ has class at most 4 .

\subsection{The subgroup $\left\langle a, a^{x}, a^{y}\right\rangle$}

We let $G=\langle a, b, c\rangle$ where $b=a^{x}$ and $c=a^{y}$, and we show that $G$ is nilpotent. In proving that $G$ is nilpotent we may assume that the Hirsch-Plotkin radical of $G$ is trivial, so that the centre of $G$ is trivial, and so that $G$ has no elements of order 2, 3 or 5 . Using the results of Section 5.4 we may assume that $\left\langle a, b, a^{c}\right\rangle$ is nilpotent of class at most 4 , with similar results for any permutation of $a, b, c$.

Step 1: Show that the subgroup $H=\langle a,[a, b, b], c\rangle$ is nilpotent of class at most 3 . 
We have

$$
[[b, a, a],[c, a, a]]=[[b, a, a],[c, a, c]]=1
$$

since both these elements lie in $\gamma_{5}\left\langle a, c, a^{b}\right\rangle=\{1\}$. Using Lemma 5 (with $b$ and $c$ interchanged) this implies

$$
\left[[b, a, b]^{8},[c, a, a]\right]=\left[[b, a, b]^{8},[c, a, c]\right]=1 .
$$

Applying Traustason's theorem to the 2-generator groups $\langle[b, a, b],[c, a, a]\rangle$ and $\langle[b, a, b],[c, a, c]\rangle$ we see that $[[b, a, b],[c, a, a]]$ and $[[b, a, b],[c, a, c]]$ are 2-elements, and so

$$
[[b, a, b],[c, a, a]]=[[b, a, b],[c, a, c]]=1 .
$$

This implies that $[c, a, a]$ and $[c, a, c] \in \zeta(H)$. Also

$$
[[a, b, b], c,[a, b, b]]=[[a, b, b], c, c, c]=1
$$

since both these elements lie in $\gamma_{5}\left\langle b, c, b^{a}\right\rangle=\{1\}$. So $H / \zeta(H)$ is is a homomorphic image of $T$, and is nilpotent, and $H$ is nilpotent. The nilpotent quotient algorithm shows that the class of $H$ is at most 4 .

To show that the class is at most 3 we need one more relation. We have

$$
[[b, a, a],[c, a], c] \in \gamma_{5}\left\langle a, c, a^{b}\right\rangle=\{1\}
$$

and we use Lemma 5 to show that this implies that

$$
[[a, b, b],[c, a], c]=1 .
$$

We have $b=a^{x}$, and we let $d=a^{x^{2}}, e=a^{x^{-1}}$. By Lemma 5 the subgroup $\langle a, b, d, e\rangle$ is nilpotent of class at most 3 . And if $u$ is any one of $b, d, e$ then $\gamma_{3}\langle[u, a, a],[c, a]\rangle \leq$ $\gamma_{5}\left\langle a, u, a^{c}\right\rangle=\{1\}$. Also, if $u, v$ are any two of $b, d, e$ then

$$
[[c, a],[u, a, a][v, a, a],[u, a, a][v, a, a]]=[[c, a],[u v, a, a],[u v, a, a]] \in \gamma_{5}\left\langle a, a^{u v}, a^{c}\right\rangle=1 .
$$

Via the argument in Section 5.2, these relations imply that $\langle[b, a, a],[d, a, a],[e, a, a],[c, a]\rangle$ is nilpotent of class at most 2 , so that Lemma 5 implies that

$$
\left[[b, a, b]^{8},[c, a]\right]=[[b, a, a],[c, a]]^{-34}[[d, a, a],[c, a]]^{6}[[e, a, a],[c, a]]^{-30} .
$$

Now we showed above that $[[b, a, a],[c, a], c]=1$, and this implies that $[[d, a, a],[c, a], c]=$ 1 and $[[e, a, a],[c, a], c]=1$. So

$$
\left[[b, a, b]^{8},[c, a], c\right]=1 .
$$

But $[b, a, b]=[a, b, b]^{-1}$, and the class of $\langle a,[a, b, b], c\rangle$ is at most 4 , so

$$
[[a, b, b],[c, a], c]^{8}=1
$$

and hence

$$
[[a, b, b],[c, a], c]=1
$$


as claimed. Adding this relation to $H$, the nilpotent quotient algorithm shows that $H$ has class at most 3. This completes Step 1.

Step 2: Show $[a, b, b]=1$.

Since $H=\langle a,[a, b, b], c\rangle$ is nilpotent of class at most 3 , we have

$$
[a, b, b, c, a, a]=[a, b, b, c, a, c]=1 .
$$

We also have $[a, b, b, c, a]=[a, b, b,[c, a]]$ so that

$$
[a, b, b, c, a, b]=[a, b, b,[c, a], b] \in \gamma_{5}\left\langle a, b, a^{c}\right\rangle=\{1\} .
$$

So $[a, b, b, c, a]$ is central in $G$, and therefore trivial.

Next, using the fact that $\left\langle b, c, b^{a}\right\rangle$ has class at most 4 we have

$$
[a, b, b, c, b, b]=[a, b, b, c, b, c]=1
$$

and

$$
[a, b, b, c, b, a]=[a, b, b,[c, b], a] \in \gamma_{5}\left\langle a, b, b^{c}\right\rangle=\{1\},
$$

so that $[a, b, b, c, b]=1$.

And finally, using the fact that $H$ has class at most 3 again, we have

$$
[a, b, b, c, c, a]=[a, b, b, c, c, c]=1
$$

and since $\left\langle b, c, b^{a}\right\rangle$ has class at most 4 we have

$$
[a, b, b, c, c, b]=1 \text {. }
$$

So $[a, b, b, c, c]$ is central, and therefore trivial.

We now have

$$
[a, b, b, c, a]=[a, b, b, c, b]=[a, b, b, c, c]=1
$$

and so $[a, b, b, c]=1$ which implies that $[a, b, b]=1$. This completes Step 2 .

So we have shown that $[a, b, b]=1$, and by symmetry we have

$$
[b, a, a]=[b, a, b]=[c, a, a]=[c, a, c]=[c, b, b]=[c, b, c]=1 .
$$

Step 3: Show that $[a, b]=1$.

We know that the subgroups $\left\langle b, b^{a}, c\right\rangle$ and $\left\langle a, a^{b}, c\right\rangle$ are nilpotent of class at most 4. This implies that $\langle a,[a, b], c\rangle$ and $\langle b,[a, b], c\rangle$ are nilpotent of class at most 4 . It follows that $[c,[a, b],[a, b],[a, b]],[c,[a, b],[a, b], c]$ and $[c,[a, b], c, c]$ all lie in the centre of $G$, and so are all trivial. We also have

$$
\begin{aligned}
1 & =\left[c, a^{b}, a^{b}, a^{b}\right] \\
& =[c, a[a, b], a[a, b], a[a, b]] \\
& =[c,[a, b],[a, b], a]^{3}[c,[a, b],[a, b],[a, b]]
\end{aligned}
$$


using the fact that $\langle a,[a, b], c\rangle$ has class at most 4 and the fact that $[c, a, a]=[b, a, a]=$ 1. So $[c,[a, b],[a, b], a]=1$. Similarly, $[c,[a, b],[a, b], b]=1$. So

$$
[c,[a, b],[a, b], a]=[c,[a, b],[a, b], b]=[c,[a, b],[a, b], c]=1
$$

and hence $[c,[a, b],[a, b]]=1$.

Using the fact that $\left\langle a, a^{b}, c\right\rangle$ has class at most 4 , we have

$$
\begin{aligned}
1 & =\left[c, a^{b}, a^{b}, c\right] \\
& =[c, a[a, b], a[a, b], c] \\
& =[c, a, a, c][c, a,[a, b], c]^{2}[c,[a, b],[a, b], c]
\end{aligned}
$$

and so $[c, a,[a, b], c]=1$. Since $[[c, a],[b, a]]=[c, a,[a, b]]^{-1}$, this gives $[[c, a],[b, a], c]=$ 1 , and by symmetry $[[c, a],[b, a], b]=1$. Now $[b, a]$ and $[c, a]$ both commute with $a$ by Step 2 , and so $[[c, a],[b, a]]$ is in the centre of $G$, and therefore trivial. Since $[c, a]$ commutes with $c$ by Step 2, we also have $[[a, b], c,[c, a]]=1$, and so

$$
\begin{aligned}
{[a, b, c]^{a} } & =[[a, b, c[c, a]] \\
& =[a, b,[c, a]][a, b, c][a, b, c,[c, a]] \\
& =[a, b, c]
\end{aligned}
$$

So $[a, b, c, a]=1$. By symmetry we have $[[c, b],[b, a]]=1$, which similarly gives $[a, b, c, b]=1$. Using the fact that $\langle a,[a, b], c\rangle$ has class at most 4 again, we have

$$
[a, b,[c, a], c]=[a, b, c,[c, a]]=[a, b, c, c, a][a, b, c, a, c]^{-1}
$$

and so $[a, b, c, c, a]=1$. Similarly, $[a, b, c, c, b]=1$, and since (as we showed above) $[a, b, c, c, c]=1$ we have $[a, b, c, c]=1$.

So $[a, b, c]$ is central, and therefore trival, and since $[a, b, a]=[a, b, b]=1$ this implies that $[a, b]=1$. This completes Step 3.

So $[a, b]=1$, and by symmetry we have $[a, c]=[b, c]=1$ also, so that $G$ is abelian.

So the assumption that the Hirsch-Plotkin radical of $G$ is trivial leads to the conclusion that $G$ is abelian. This proves that if we lift the assumption that the HirschPlotkin radical of $G$ is trivial we can still conclude that $G$ is nilpotent.

So $G$ is a nilpotent 4-Engel group generated by elements $a, b, c$ with the property that any two of $a, b, c, a^{b}, b^{a}, a^{c}, c^{a}, b^{c}, c^{b}$ generate a subgroup of class at most 3 . Using these properties, the nilpotent quotient algorithm shows that $G$ is nilpotent of class at most 6 and that $\gamma_{6}(G)$ has exponent dividing 10. It also shows that if $N$ is the normal closure in $G$ of one of the generators $a, b, c$, then $\gamma_{3}(N)$ has exponent dividing 60 .

Using the fact that $G$ has no $\{2,3,5\}$-elements we conclude that $G$ is nilpotent of class at most 5 , and that the normal closure in $G$ of any of the generators $a, b, c$ is nilpotent of class at most 2 . 


\section{A key result}

The results of Section 5 on the subgroups generated by three conjugates enable us to prove the following key result.

Lemma 6. Let $E$ be a 4-Engel group with trivial Hirsch-Plotkin radical. If a, $x, c \in E$ then

$$
[c,[x, a, a, a],[x, a, a, a],[x, a, a, a]]=1 .
$$

Proof. In this proof we make use of several commutator identities which hold in the free 4-Engel group of rank 2. These identities are all easy to check with the nilpotent quotient algorithm. Alternatively, they can even be "hand checked", working from a PCP for the free 4-Engel group of rank 2.

We let $G$ be the group generated by $a, a^{x}$, and $c$, and we let $b=a^{a^{a^{x}}}$. Note that $[a, b]=1$, and $a^{-1} b=[a,[a,[a, x]]]=[x, a, a, a]^{-1}$. We also have $b=a\left[a^{x}, a, a\right]$, so that $b$ lies in the normal closure of $a$ in the subgroup $\left\langle a, a^{x}\right\rangle$.

Now let $r \in \mathbb{Z}$, and let $u, v, w \in\{a, b\}$. Consider the commutator $\left[c^{r}, a, u, v, w\right]$. We have

$$
\left[c^{r}, a, u, v, w\right]=\left[a^{-c^{r}} a, u, v, w\right] \in\left\langle a, a^{x}, a^{c^{r}}\right\rangle .
$$

The results of Section 5 imply that the normal closure of $a$ in $\left\langle a, a^{x}, a^{c^{r}}\right\rangle$ is nilpotent of class at most 2 , and this implies that $\left[c^{r}, a, u, v, w\right]=1$. Similarly $\left[c^{r}, b, u, v, w\right]=1$ for all $u, v, w \in\{a, b\}$. We also have

$$
\left[c^{r}, a, a, a^{-1} b\right]=\left[c^{r}, a, b, a^{-1} b\right]=\left[c^{r}, b, a, a^{-1} b\right]=\left[c^{r}, b, b, a^{-1} b\right]=1
$$

for all $r \in \mathbb{Z}$. We consider the group $H$ generated by $a, b, c$, and we show that these identities imply that $H$ is nilpotent. As usual, in proving that $H$ is nilpotent we can assume that the Hirsch-Plotkin radical of $H$ is trivial, so that $H$ has trivial centre and has no elements of order 2 or 3 or 5 .

Step 1: Show that $\left[c^{r}, a, a\right]=\left[c^{r}, b, b\right]=1$ for all $r$.

First we show that $[c, a, a, a, c, c]=1$. From the computation of the 2-generator, 4-Engel group $\langle a, c\rangle$ we have

$$
[c, a, a, a, c, c]=[c, a, a, a]^{-3}\left[c^{2}, a, a, a\right]\left[c^{-1}, a, a, a\right]^{-1} .
$$

Now $[c, a, a, a, c, c]$ is centralized by $a$ and $c$, and $[c, a, a, a],\left[c^{2}, a, a, a\right]$ and $\left[c^{-1}, a, a, a\right]$ are all centralized by $b$. So $[c, a, a, a, c, c]$ is in the centre of $H$, which implies that $[c, a, a, a, c, c]=1$. Given that $[c, a, a, a, c, c]=1$ we have

$$
[c, a, a, a, c]^{4}=[c, a, a, a]^{-8}\left[c^{-1}, a, a, a\right]^{-8} .
$$

The right hand side of this equation is centralized by $a$ and $b$, and (as we have just shown) the left hand side is centralized by $c$. So $[c, a, a, a, c]^{4}=1$ and hence $[c, a, a, a, c]=1$. Since

$$
[c, a, a, a, a]=[c, a, a, a, b]=1
$$


this implies that $[c, a, a, a]=1$. If $[c, a, a, a]=1$ then $\left[c^{2}, a, a, a\right]$ and $\left[c^{-1}, a, a, a\right]$ have order dividing 8 , so we also have

$$
\left[c^{2}, a, a, a\right]=\left[c^{-1}, a, a, a\right]=1 .
$$

This implies that the normal closure of $a$ in $\langle a, c\rangle$ has class at most 2 , so that $\left[c^{r}, a, a, a\right]=1$ for all $r$. Note that since $\left[c^{r}, a, a, a^{-1} b\right]=1$ this implies that $\left[c^{r}, a, a, b\right]=$ 1 for all $r$.

Next, using the fact that $\left[c^{r}, a, a, a\right]=1$ for $r=1,2,-1$ we obtain

$$
[c, a, a, c, c]^{2}=[c, a, a]^{3}\left[c^{2}, a, a\right]^{-1}\left[c^{-1}, a, a\right]
$$

and so $[c, a, a, c, c]=1$. This gives

$$
[c, a, a, c]=[c, a, a]^{-2}\left[c^{2}, a, a\right]
$$

and so $[c, a, a, c]=1$. This in turn gives $[c, a, a]=1$, which implies that $\left[c^{r}, a, a\right]=1$ for all $r$.

So we have $\left[c^{r}, a, a\right]=1$ for all $r$, and similarly we can show that $\left[c^{r}, b, b\right]=1$ for all $r$.

Step 2: Show that $\left\langle a, b, a^{c}, b^{c}\right\rangle$ has class at most 2 .

If we consider the group $\left\langle a, b, a^{c}, b^{c}\right\rangle$ we see that $a$ commutes with $b$ and $a^{c}$ and that $b$ commutes with $a$ and $b^{c}$. So

$$
\left\langle a, b, a^{c}, b^{c}\right\rangle=\left\langle a, b^{c}\right\rangle \times\left\langle b, a^{c}\right\rangle .
$$

Now

$$
1=\left[c, b, a, a^{-1} b\right]=[c, b, a, b]\left[c, b, a, a^{-1}\right]^{b} .
$$

We have $[c, b, a, b]=1$ since $[c, b, b]=[a, b]=1$, and so $\left[c, b, a, a^{-1}\right]=1$. This implies that $[c, b, a, a]=1$ and hence that $\left[b^{-c}, a, a\right]=1$. Similarly, $\left[c^{-1}, a, b, a^{-1} b\right]=1$ gives $\left[a^{-c^{-1}}, b, b\right]=1$ which, by conjugating with $c$, gives $\left[a^{-1}, b^{c}, b^{c}\right]=1$. So $\left\langle a, b^{c}\right\rangle$ is nilpotent of class at most 2. Similarly $\left\langle b, a^{c}\right\rangle$ is nilpotent of class at most 2 , and so $\left\langle a, b, a^{c}, b^{c}\right\rangle=\left\langle a, b^{c}\right\rangle \times\left\langle b, a^{c}\right\rangle$ is nilpotent of class at most 2 .

Step 3: Show that $H=\langle a, b, c\rangle$ is trivial (assuming that the Hirsch-Plotkin radical of $H$ is trivial).

Since $\left[c, a^{-1} b\right] \in\left\langle a, b, a^{c}, b^{c}\right\rangle$ we have

$$
\left[c, a^{-1} b, a^{-1} b, a^{-1} b\right]=\left[c, a^{-1} b, a^{-1} b, a\right]=\left[c, a^{-1} b, a^{-1} b, b\right]=1 .
$$

By considering the group $\left\langle a, b, a^{c^{r}}, b^{c^{r}}\right\rangle$, we similarly obtain

$$
\left[c^{r}, a^{-1} b, a^{-1} b, a^{-1} b\right]=\left[c^{r}, a^{-1} b, a^{-1} b, a\right]=\left[c^{r}, a^{-1} b, a^{-1} b, b\right]=1
$$

for all $r$.

So, as above, we have

$$
\left[c, a^{-1} b, a^{-1} b, c, c\right]^{2}=\left[c, a^{-1} b, a^{-1} b\right]^{3}\left[c^{2}, a^{-1} b, a^{-1} b\right]^{-1}\left[c^{-1}, a^{-1} b, a^{-1} b\right]
$$


so that $\left[c, a^{-1} b, a^{-1} b, c, c\right]=1$. This gives

$$
\left[c, a^{-1} b, a^{-1} b, c\right]=\left[c, a^{-1} b, a^{-1} b\right]^{-2}\left[c^{2}, a^{-1} b, a^{-1} b\right]
$$

and so $\left[c, a^{-1} b, a^{-1} b, c\right]=1$. This in turn gives $\left[c, a^{-1} b, a^{-1} b\right]=1$, which implies that $\left[c^{r}, a^{-1} b, a^{-1} b\right]=1$ for all $r$.

Using the fact that $\left\langle a, b, a^{c}, b^{c}\right\rangle$ has class at most 2 , and the fact that $[c, a, a]=$ $[c, b, b]=1$ we see that

$$
\begin{aligned}
1 & =\left[c, a^{-1} b, a^{-1} b\right] \\
& =\left[[c, b]\left[c, a^{-1}\right], a^{-1} b\right] \\
& =\left[c, b, a^{-1}\right]\left[c, a^{-1}, b\right] \\
& =[c, b, a]^{-1}[c, a, b]^{-1} .
\end{aligned}
$$

We also have

$$
\begin{aligned}
& {[c, a b]=[c, b][c, a][c, a, b]} \\
& {[c, b a]=[c, a][c, b][c, b, a]}
\end{aligned}
$$

Since $a b=b a$ this implies that

$$
[[c, b],[c, a]]=[c, b, a][c, a, b]^{-1}=[c, a, b]^{-2}=[c, b, a]^{2} .
$$

We consider the subgroup $N=\langle c,[c, a],[c, b],[c, a, b]\rangle \leq H$. Since $[c, b, a]=$ $[c, a, b]^{-1}$ and $[c, a, b, a]=[c, a, b, b]=1, N \triangleleft H$. This implies that the centre of $N$ is a normal subgroup of $H$, and so is contained in the Hirsch-Plotkin radical of $H$. We are assuming (for the moment) that the Hirsch-Plotkin radical of $H$ is trivial, and so $N$ has trivial centre. We show that this implies that $N=\{1\}$. But if $N$ is trivial, then $H$ is abelian, and therefore also trivial.

First consider the subgroup $K=\langle c,[c, a],[c, b]\rangle \leq N$. Since $K=\left\langle c, c^{a}, c^{b}\right\rangle, K$ is nilpotent. Since $\left\langle a, b, a^{c}, b^{c}\right\rangle$ has class at most 2 , we also have

$$
[[c, a],[c, b],[c, a]]=[[c, a],[c, b],[c, b]]=1,
$$

which can be expressed in the form

$$
\left[c^{-1} c^{a}, c^{-1} c^{b}, c^{-1} c^{a}\right]=\left[c^{-1} c^{a}, c^{-1} c^{b}, c^{-1} c^{b}\right]=1 .
$$

In addition, if we let $u=c, v=c^{a}, w=c^{b}$ then any two of $u, v, w, u^{v}, v^{u}, u^{w}, w^{u}, v^{w}, w^{v}$ generate a subgroup of class at most 3 . The nilpotent quotient algorithm using these relations (together with the 4 -Engel identity) shows that $K$ is nilpotent of class at most 4. (The nilpotent quotient algorithm actually shows that $K$ has class at most 5, with $\gamma_{5}(K)$ of exponent 8 . But we are assuming that $H$ has no elements of order 2.)

Now we make use of the fact that $[c, a, b]^{-2}=[[c, b],[c, a]]$. This implies that

$$
\left[[c, a, b]^{2},[c, a]\right]=\left[[c, a, b]^{2},[c, b]\right]=1,
$$


which (using the fact that $H$ has no elements of order 2) implies that

$$
[[c, a, b],[c, a]]=[[c, a, b],[c, b]]=1
$$

Next, we have

$$
\left[[c, a, b]^{2}, c,[c, a, b]^{2}\right]=1
$$

which implies that

$$
[[c, a, b], c,[c, a, b]]^{8}=1
$$

and hence that

$$
[[c, a, b], c,[c, a, b]]=1 .
$$

We also have $\left[[c, a, b]^{2}, c, c, c\right]=1$ which similarly implies that $[[c, a, b], c, c, c]=1$, and so the subgroup $\langle[c, a, b], c\rangle$ has class at most 3 .

In particular, $\left[[c, a, b]^{2}, c, c\right]$ commutes with $[c, a, b]$. Also $\left[[c, a, b]^{2}, c, c\right] \in \gamma_{4}(K)$, and since $K$ has class at most 4 , this implies that $\left[[c, a, b]^{2}, c, c\right]$ commutes with $c$, $[c, a]$ and $[c, b]$. So $\left[[c, a, b]^{2}, c, c\right]$ lies in the centre of $N$. But $N$ has trivial centre, and so we have $\left[[c, a, b]^{2}, c, c\right]=1$.

Similarly, $\left[[c, a, b]^{2}, c,[c, a]\right]$ commutes with $c,[c, a]$ and $[c, b]$. And so since $[c, a]$ and $\left[[c, a, b]^{2}, c\right]$ both commute with $[c, a, b]$ we see that $\left[[c, a, b]^{2}, c,[c, a]\right]$ lies in the centre of $N$, and is trivial. By symmetry $\left[[c, a, b]^{2}, c,[c, b]\right]=1$.

So $\left[[c, a, b]^{2}, c\right]$ commutes with $c,[c, a]$ and $[c, b]$. It also commutes with $[c, a, b]$, and so $\left[[c, a, b]^{2}, c\right]$ is in the centre of $N$, and is trivial. This in turn implies that $[c, a, b]^{2}$ is in the centre of $N$, and hence that $[c, a, b]=1$.

But if $[c, a, b]=1$ then $N=K$, and since $K$ is nilpotent this implies that $N$ is nilpotent. But $N$ has trivial centre, and so $N=\{1\}$, and hence $H=\{1\}$. This completes Step 3.

So the assumption that the Hirsch-Plotkin radical of $H$ is trivial leads to the conclusion that $H=\{1\}$. It follows that $H$ is nilpotent. The nilpotent quotient algorithm, using the 4-Engel identity and the relations

$$
[c, a, u, v, w]=[c, b, u, v, w]=1
$$

for all $u, v, w \in\{a, b\}$, together with the relations

$$
\left[c, a, a, a^{-1} b\right]=\left[c, a, b, a^{-1} b\right]=\left[c, b, a, a^{-1} b\right]=\left[c, b, b, a^{-1} b\right]=1
$$

shows that

$$
\left[c, a b^{-1}, a b^{-1}, a b^{-1}\right]=1
$$

and hence that

$$
[c,[x, a, a, a],[x, a, a, a],[x, a, a, a]]=1 .
$$




\section{Proof of Theorem 1}

Having established Lemma 6 we are at last in a position to prove that 4-Engel groups are locally nilpotent. Let $G$ be a 4 -Engel group with trivial Hirsch-Plotkin radical, let $y, z \in G$, and let $a=[y, z, z, z]$. Then Lemma 6 implies that if $x \in G$ then

$$
\left[a^{x}, a, a\right]=[[a, x], a, a]=[x, a, a, a]^{-1}=1 .
$$

Substituting $x^{-1}$ for $x$, and then conjugating by $x$, we also obtain $\left[a, a^{x}, a^{x}\right]=1$. So the subgroup $\left\langle a, a^{x}\right\rangle$ is nilpotent of class at most 2. This implies that if $a_{1}, a_{2}$ are any two conjugates of $a$ then the subgroup $\left\langle a_{1}, a_{2}\right\rangle$ is nilpotent of class at most 2 . So Lemma 7 below implies that the normal closure of $a$ in $G$ is locally nilpotent. This implies that $a=[y, z, z, z]$ lies in the Hirsch-Plotkin radical of $G$, and hence that $[y, z, z, z]=1$. This is true for all $y, z \in G$, and so $G$ is a 3-Engel group, and therefore locally nilpotent. The assumption that the Hirsch-Plotkin radical of $G$ is trivial then implies that $G=\{1\}$. So the following lemma completes the proof of Theorem 1 .

Lemma 7. Let $G$ be a 4-Engel group with trivial Hirsch-Plotkin radical, and let $a \in G$. Suppose that if $a_{1}, a_{2}$ are any two conjugates of a in $G$, then $\left\langle a_{1}, a_{2}\right\rangle$ has class at most 2. Then the normal closure of a in $G$ is locally nilpotent.

Proof. Let $a_{1}, a_{2}, \ldots, a_{k}$ be conjugates of $a$, and let $H=\left\langle a_{1}, a_{2}, \ldots, a_{k}\right\rangle$. We show, by induction on $k$, that $H$ is nilpotent of class at most $k$, and that the normal closure of $a_{i}$ in $H$ is abelian for $i=1,2, \ldots, k$. This proves the lemma.

The case $k=2$ is the hypothesis of the lemma, so we start with the case $k=3$. As a first step, we consider the case when $a_{1}$ and $a_{2}$ commute. Then $H$ is a 4-Engel group, and

$$
\left[a_{1}, a_{2}\right]=\left[a_{3}, a_{1}, a_{1}\right]=\left[a_{3}, a_{1}, a_{3}\right]=\left[a_{3}, a_{2}, a_{2}\right]=\left[a_{3}, a_{2}, a_{3}\right]=1 .
$$

Since $a_{1}, a_{2}$ and $a_{3}$ are conjugates the results of Section 5 imply that $H$ is nilpotent. Given that $H$ is nilpotent, these relations imply that $H$ is nilpotent of class at most 3 , and that the normal closure of $a_{i}$ in $H$ is abelian for $i=1,2,3$. (This calculation relies on the assumption that $H$ contains no elements of order 2.)

Next we consider the general case $H=\left\langle a_{1}, a_{2}, a_{3}\right\rangle$, without the assumption that $\left[a_{1}, a_{2}\right]=1$. Note that the fact that $\left[a_{1}, a_{2}, a_{1}\right]=1$ implies that $a_{1}$ commutes with $a_{1}^{a_{2}}$. So, by the case dealt with above, $\left\langle a_{1}, a_{1}^{a_{2}}, a_{3}\right\rangle$ is nilpotent of class at most 3 , and the normal closure of $a_{3}$ in $\left\langle a_{1}, a_{1}^{a_{2}}, a_{3}\right\rangle$ is abelian. this implies that

$$
\left[a_{1}, a_{2}, a_{3}, a_{3}\right]=\left[a_{1}^{-1} a_{1}^{a_{2}}, a_{3}, a_{3}\right]=1 .
$$

Similarly $\left[a_{i}, a_{j}, a_{k}, a_{k}\right]=1$ for all $i, j, k \in\{1,2,3\}$. It follows that $H$ satisfies the following relations:

$$
\begin{aligned}
{\left[a_{i}, a_{j}, a_{j}\right] } & =1 \quad \text { for all } i, j \in\{1,2,3\} \\
{\left[a_{i}, a_{j}, a_{k}, a_{k}\right] } & =1 \quad \text { for all } i, j, k \in\{1,2,3\} .
\end{aligned}
$$

Since $H$ is generated by three conjugates, $H$ is nilpotent. These relations then imply that $H$ is nilpotent of class at most 3 and that the normal closure of $a_{i}$ in $H$ is abelian for $i=1,2,3$. 
The case $k=3$ enables us to deduce the following identities, which hold for any conjugates $a_{1}, a_{2}, \ldots, a_{k}$ of $a$ for any $k \geq 3$.

$$
\begin{gathered}
{\left[a_{1}, a_{2}, \ldots, a_{k-1}, a_{k}, a_{k}\right]=1} \\
{\left[a_{k},\left[a_{1}, a_{2}, \ldots, a_{k-1}\right],\left[a_{1}, a_{2}, \ldots, a_{k-1}\right],\left[a_{1}, a_{2}, \ldots, a_{k-1}\right]\right]=1 .}
\end{gathered}
$$

To establish these identities, we let $u=\left[a_{1}, a_{2}, \ldots, a_{k-2}\right]$, and let $b=a_{k-1}^{u}$. Then $b, a_{k-1}, a_{k}$ are all conjugates of $a$, and so by the case $k=3$ we see that the group $\left\langle b, a_{k-1}, a_{k}\right\rangle$ is nilpotent of class at most 3 and the normal closure of any of $b, a_{k-1}, a_{k}$ in $\left\langle b, a_{k-1}, a_{k}\right\rangle$ is abelian. Equations (7) and (8) follow immediately.

We now suppose that $k \geq 4$, and that the inductive hypothesis is satisfied for all smaller values of $k$. We show that if $1 \leq r<k$, then

$$
\left[a_{1}, a_{2}, \ldots, a_{k}, a_{1}\right]=\left[\left[a_{1}, a_{2}, \ldots, a_{r}\right],\left[a_{1}, a_{k}, a_{k-1}, \ldots, a_{r+1}\right]\right]^{ \pm 1} .
$$

First consider the case $r=k-1$. Let $u=a_{1}, v=\left[a_{1}, a_{2}, \ldots, a_{k-1}\right], w=a_{k}$. Then $[u, v]=1$ by the inductive hypothesis, $[w, u, u]=[w, u, w]=1$, and $[v, w, w]=$ $[w, v, v, v]=1$ by Equations (7) and (8). So $\langle u, v, w\rangle$ is a homomorphic image of $T$, and hence nilpotent of class at most 4 . The nilpotent quotient algorithm shows that $[v, w, u]=[v,[u, w]]^{-1}$, which establishes Equation (9) for the case $r=k-1$. Reversing the order of $a_{2}, a_{3}, \ldots, a_{k}$, the case $r=k-1$ also gives

$$
\left[a_{1},\left[a_{1}, a_{k}, a_{k-1}, \ldots, a_{3}, a_{2}\right]\right]=\left[\left[a_{1}, a_{2}\right],\left[a_{1}, a_{k}, a_{k-1}, \ldots, a_{3}\right]\right]^{-1}
$$

since

$$
\left[a_{1},\left[a_{1}, a_{k}, a_{k-1}, \ldots, a_{3}, a_{2}\right]\right]=\left[a_{1}, a_{k}, a_{k-1}, \ldots, a_{3}, a_{2}, a_{1}\right]^{-1}
$$

and

$$
\left[\left[a_{1}, a_{2}\right],\left[a_{1}, a_{k}, a_{k-1}, \ldots, a_{3}\right]\right]=\left[\left[a_{1}, a_{k}, a_{k-1}, \ldots, a_{3}\right],\left[a_{1}, a_{2}\right]\right]^{-1} .
$$

So, to establish Equation (9), it is sufficient to show that if $2 \leq r \leq k-2$, then

$$
\left[\left[a_{1}, a_{2}, \ldots, a_{r}\right],\left[a_{1}, a_{k}, a_{k-1}, \ldots, a_{r+1}\right]\right]=\left[\left[a_{1}, a_{2}, \ldots, a_{r+1}\right],\left[a_{1}, a_{k}, a_{k-1}, \ldots, a_{r+2}\right]\right]^{-1} .
$$

Let $u=\left[a_{1}, a_{2}, \ldots, a_{r}\right], v=\left[a_{1}, a_{k}, a_{k-1}, \ldots, a_{r+2}\right], w=a_{r+1}$. Then, using the inductive hypothesis, $[u, v]=1,[w, u, u]=[w, u, w]=1$, and $[w, v, v]=[w, v, w]=1$. So, as above, $[u,[v, w]]=[u, w, v]^{-1}$. This establishes Equation (9).

Now consider a commutator $c=\left[b_{1}, b_{2}, \ldots, b_{k+1}\right]$ where $b_{1}, b_{2}, \ldots, b_{k+1}$ lie in the set $\left\{a_{1}, a_{2}, \ldots, a_{k}\right\}$. We show that $c \in \zeta(H)$. By induction, $c=1$ unless $\left\{b_{1}, b_{2}, \ldots, b_{k}\right\}=$ $\left\{a_{1}, a_{2}, \ldots, a_{k}\right\}$. Also, by Equation (7), $c=1$ if $b_{k}=b_{k+1}$. So there is no loss in generality in assuming that $b_{k+1}=a_{1}, b_{k}=a_{k}$, and that $\left\{b_{1}, b_{2}, \ldots, b_{k-1}\right\}=$ $\left\{a_{1}, a_{2}, \ldots, a_{k-1}\right\}$. Then, using the inductive hypothesis, we see that $\left[b_{1}, b_{2}, \ldots, b_{k-1}\right]$ can be expressed as a product $u_{1} u_{2} \ldots u_{r}$, where each $u_{i}$ is a commutator of the form $\left[a_{1}, a_{\sigma(2)}, a_{\sigma(3)}, \ldots, a_{\sigma(k-1)}\right]$ for some permutation $\sigma$ of $\{2,3, \ldots, k-1\}$. So

$$
c=\left[b_{1}, b_{2}, \ldots, b_{k+1}\right]=\left[u_{1} u_{2} \ldots u_{r}, a_{k}, a_{1}\right]=\left[\prod_{i=1}^{r}\left[u_{i}, a_{k}\right]^{u_{i+1} u_{i+2} \ldots u_{r}}, a_{1}\right] .
$$


Now the inductive hypothesis implies that $u_{1}, u_{2}, \ldots, u_{r}$ commute with $a_{1}$. So $c$ is a product of conjugates of the commutators $\left[u_{1}, a_{k}, a_{1}\right],\left[u_{2}, a_{k}, a_{1}\right], \ldots,\left[u_{r}, a_{k}, a_{1}\right]$. So to show that $c \in \zeta(H)$ it is sufficient to show that $\left[u_{i}, a_{k}, a_{1}\right] \in \zeta(H)$ for $i=1,2, \ldots, r$. Clearly, it is sufficient to show that $\left[a_{1}, a_{2}, \ldots, a_{k}, a_{1}\right] \in \zeta(H)$.

So consider $d=\left[a_{1}, a_{2}, \ldots, a_{k}, a_{1}, a_{i}\right]$, where $1 \leq i \leq k$. If $i=1$, then $d=1$ by Equation (7). If $i=k$, let $u=a_{1}, v=\left[a_{1}, a_{2}, \ldots, a_{k-1}\right], w=a_{k}$. Then $[u, v]=1$, $[w, u, u]=[w, u, w]=1$, and $[v, w, w]=[w, v, v, v]=1$. So, as above, $\langle u, v, w\rangle$ is nilpotent of class at most 4 , and nilpotent quotient algorithm shows that $[v, w, u, w]=$ 1. So $d=1$ if $i=k$.

Now let $1<i<k$. Then, by Equation (9),

$$
d=\left[a_{1}, a_{2}, \ldots, a_{k}, a_{1}, a_{i}\right]=\left[\left[\left[a_{1}, a_{2}, \ldots, a_{i}\right],\left[a_{1}, a_{k}, a_{k-1}, \ldots, a_{i+1}\right]\right]^{ \pm 1}, a_{i}\right] .
$$

So to show that $d=1$, we need to show that

$$
\left[\left[a_{1}, a_{2}, \ldots, a_{i}\right],\left[a_{1}, a_{k}, a_{k-1}, \ldots, a_{i+1}\right], a_{i}\right]=1 .
$$

Let $u=\left[a_{1}, a_{2}, \ldots, a_{i-1}\right], v=\left[a_{1}, a_{k}, \ldots, a_{i+1}\right], w=a_{i}$. Then $[u, v]=1,[w, u, u]=$ $[w, u, w]=1$, and $[v, w, w]=[w, v, v, v]=1$. (If $i>2$ we also have $[v, w, v]=1$, but we do not need this relation.) So $\langle u, v, w\rangle$ is nilpotent, and the nilpotent quotient algorithm shows that $[u, w, v, w]=1$. So $d=1$ as required.

This shows that $\left[a_{1}, a_{2}, \ldots, a_{k}, a_{1}\right] \in \zeta(H)$, and hence that $\left[b_{1}, b_{2}, \ldots, b_{k+1}\right] \in \zeta(H)$ for all $b_{1}, b_{2}, \ldots, b_{k+1} \in\left\{a_{1}, a_{2}, \ldots, a_{k}\right\}$. So $H$ is nilpotent of class at most $k+1$. To complete the proof of Lemma 7 we need to show that $\left[a_{1}, a_{2}, \ldots, a_{k}, a_{1}\right]=1$, since by the argument we have just used, this will imply that $\left[b_{1}, b_{2}, \ldots, b_{k+1}\right]=1$ for all $b_{1}, b_{2}, \ldots, b_{k+1} \in\left\{a_{1}, a_{2}, \ldots, a_{k}\right\}$.

First we show that $\left[a_{1}, a_{2}, \ldots, a_{k-3},\left[a_{k-2}, a_{k-1}\right], a_{k}, a_{1}\right]=1$. This relation holds because if we let $a_{k-2}=b, a_{k-1}=c$, then $\left[a_{k-2}, a_{k-1}\right]=[b, c]=b^{-1} b^{c}$, so that

$$
\begin{aligned}
& {\left[a_{1}, a_{2}, \ldots, a_{k-3},\left[a_{k-2}, a_{k-1}\right]\right]} \\
& \quad=\left[a_{1}, a_{2}, \ldots, a_{k-3}, b^{-1} b^{c}\right] \\
& \quad=\left[a_{1}, a_{2}, \ldots, a_{k-3}, b^{c}\right]\left[a_{1}, a_{2}, \ldots, a_{k-3}, b^{-1}\right]\left[a_{1}, a_{2}, \ldots, a_{k-3}, b^{-1}, b^{c}\right] \\
& \quad=\left[a_{1}, a_{2}, \ldots, a_{k-3}, b^{c}\right]\left[a_{1}, a_{2}, \ldots, a_{k-3}, b^{-1}\right] \quad \text { by induction. }
\end{aligned}
$$

It follows that

$$
\begin{aligned}
& {\left[a_{1}, a_{2}, \ldots, a_{k-3},\left[a_{k-2}, a_{k-1}\right], a_{k}, a_{1}\right]} \\
& \quad=\left[a_{1}, a_{2}, \ldots, a_{k-3},\left[a_{k-2}, a_{k-1}\right],\left[a_{1}, a_{k}\right]\right]^{-1} \\
& \quad=\left[\left[a_{1}, a_{2}, \ldots, a_{k-3}, b^{c}\right]\left[a_{1}, a_{2}, \ldots, a_{k-3}, b^{-1}\right],\left[a_{1}, a_{k}\right]\right]^{-1} \\
& \quad=1
\end{aligned}
$$

since, by induction,

$$
\left[\left[a_{1}, a_{2}, \ldots, a_{k-3}, b^{c}\right],\left[a_{1}, a_{k}\right]\right]=\left[\left[a_{1}, a_{2}, \ldots, a_{k-3}, b^{-1}\right],\left[a_{1}, a_{k}\right]\right]=1 .
$$


Similarly, if we let $b=a_{k}$, and let $c=\left[a_{k-2}, a_{k}\right]$, then $\left[a_{k-2}, a_{k-1}, a_{k}\right]=b^{-c} b$, and so

$$
\left[a_{1}, a_{2}, \ldots, a_{k-3},\left[a_{k-2}, a_{k-1}, a_{k}\right], a_{1}\right]=\left[a_{1}, a_{2}, \ldots, a_{k-3}, b^{-c} b, a_{1}\right]=1 .
$$

But

$$
\begin{aligned}
& {\left[a_{1}, a_{2}, \ldots, a_{k-3},\left[a_{k-2}, a_{k-1}, a_{k}\right], a_{1}\right]} \\
& \quad=\left[a_{1}, a_{2}, \ldots, a_{k-3},\left[a_{k-2}, a_{k-1}\right], a_{k}, a_{1}\right]\left[a_{1}, a_{2}, \ldots, a_{k-3}, a_{k},\left[a_{k-2}, a_{k-1}\right], a_{1}\right]^{-1},
\end{aligned}
$$

and so this implies that

$$
\left[a_{1}, a_{2}, \ldots, a_{k-3}, a_{k},\left[a_{k-2}, a_{k-1}\right], a_{1}\right]=1 \text {. }
$$

It follows that

$$
\left[a_{1}, \ldots, a_{k-3}, a_{k-2}, a_{k-1}, a_{k}, a_{1}\right]=\left[a_{1}, \ldots, a_{k-3}, a_{\sigma(k-2)}, a_{\sigma(k-1)}, a_{\sigma(k)}, a_{1}\right]
$$

for all permutations $\sigma$ of $\{k-2, k-1, k\}$.

Now let $u=x_{k-2} x_{k-1} x_{k} x_{k+1}$ and consider the following instance of the 4-Engel identity:

$$
\left[x_{1}, x_{2}, \ldots, x_{k-3}, u, u, u, u\right]=1 .
$$

If we expand the commutator $\left[x_{1}, x_{2}, \ldots, x_{k-3}, u, u, u, u\right]$, and pick out the terms which involve all the variables $x_{1}, x_{2}, \ldots, x_{k+1}$, then we obtain the identity

$$
\prod_{\sigma}\left[x_{1}, x_{2}, \ldots, x_{k-3}, x_{\sigma(k-2)}, x_{\sigma(k-1)}, x_{\sigma(k)}, x_{\sigma(k+1)}\right] \in \gamma_{k+2}\left\langle x_{1}, x_{2}, \ldots, x_{k+1}\right\rangle,
$$

where the product ranges over all permutations of $\{k-2, k-1, k, k+1\}$. If we substitute $a_{i}$ for $x_{i}$ for $i=1,2, \ldots, k$, and substitute $a_{1}$ for $x_{k+1}$, and if we use the fact that $H$ has class at most $k+1$, then we obtain

$$
\prod_{\sigma}\left[a_{1}, a_{2}, \ldots, a_{k-3}, a_{\sigma(k-2)}, a_{\sigma(k-1)}, a_{\sigma(k)}, a_{\sigma(1)}\right]=1,
$$

where the product ranges over all permutations of $\{k-2, k-1, k, 1\}$. But if $\sigma(1) \neq 1$, then by induction

$$
\left[a_{1}, a_{2}, \ldots, a_{k-3}, a_{\sigma(k-2)}, a_{\sigma(k-1)}, a_{\sigma(k)}, a_{\sigma(1)}\right]=1 .
$$

This together with Equation (10) implies that

$$
\left[a_{1}, a_{2}, \ldots, a_{k-3}, a_{k-2}, a_{k-1}, a_{k}, a_{1}\right]^{6}=1 .
$$

Since $G$ contains no elements of order 2 or 3 this implies that

$$
\left[a_{1}, a_{2}, \ldots, a_{k-3}, a_{k-2}, a_{k-1}, a_{k}, a_{1}\right]=1
$$

which completes the proof of Lemma 7. 


\section{Acknowledgements}

We are grateful to Charles Sims for his advice on using the Knuth-Bendix procedure effectively and for making available to us various versions of the Rutgers Knuth-Bendix Package, RKBP. We thank an anonymous referee for insightful comments which enabled us to significantly improve the exposition of parts of this article.

The first author was partially supported by the Australian Research Council.

\section{References}

[1] A. Abdollahi and G. Traustason, On locally finite p-groups satisfying an Engel condition, Proc. Amer. Math. Soc. 130 (2002), 2827-2836.

[2] S. Bachmuth and H.Y. Mochizuki, Third Engel groups and the MacdonaldNeumann conjecture, Bull. Austral. Math. Soc. 5 (1971), 379-386.

[3] W. Bosma, J. Cannon, and C. Playoust, The Magma algebra system I: The user language, J. Symbolic Comput. 24 (1997), 235-265.

See also http://magma.maths.usyd.edu.au/magma/

[4] W. Burnside, On groups in which every two conjugate operations are permutable, London Math. Soc. Proc. 35 (1903), 28-37.

[5] The GAP Group, GAP - Groups, Algorithms, and Programming, Version 4.4; 2004, (http://www.gap-system.org).

[6] R.H. Gilman, Presentations of groups and monoids, J. Algebra 57 (1979), 544554 .

[7] K.W. Gruenberg, The Engel elements of a soluble group, Illinois J. Math. 3 (1959), 151-168.

[8] George Havas and M.R. Vaughan-Lee, 4-Engel groups; supplementary materials (2004). Available from http://www.itee.uq.edu.au/ ${ }^{\sim}$ havas/4engel and http: //users.ox.ac.uk/ vlee/4engel

[9] H. Heineken, Engelsche Elemente der Länge drei, Illinois J. Math. 5 (1961), 681707.

[10] D.F. Holt, KBMAG (Knuth-Bendix in Monoids and Automatic Groups, Version 2.4), Software Package (2000). Available from ftp://ftp.maths.warwick.ac. $\mathrm{uk} / \mathrm{people/dfh/kbmag2/}$

[11] L.C. Kappe and W.P. Kappe, On three Engel groups, Bull. Austral. Math. Soc. 7 (1972), 391-405.

[12] F.W. Levi, Groups in which the commutator operation satisfies certain algebraic conditions, J. Indian Math. Soc. 6 (1942), 87-97. 
[13] M.F. Newman and Michael Vaughan-Lee, Engel-4 groups of exponent 5 II. Orders, Proc. London Math. Soc. (3) 79 (1999), 283-317.

[14] Werner Nickel, Computing nilpotent quotients of finitely presented groups, in Geometric and computational perspectives on infinite groups (Minneapolis, MN and New Brunswick, NJ, 1994), pages 175-191. Amer. Math. Soc., Providence, RI, 1996.

[15] Werner Nickel, Computation of nilpotent Engel groups, J. Austral. Math. Soc. (Series A) 67 (1999), 214-222.

[16] Ju. P. Razmyslov, The Hall-Higman problem, Izv. Akad. Nauk SSSR, Ser. Mat. 42 (1978), 833-847.

[17] Charles C. Sims, Verifying nilpotence, J. Symbolic Comput. 3 (1987), 231-247.

[18] C.C. Sims, Computation with finitely presented groups, Encyclopedia of mathematics 48, Cambridge University Press (1994).

[19] G. Traustason, On 4-Engel groups, J. Algebra 178 (1995), 414-429.

[20] Gunnar Traustason, Locally nilpotent 4-Engel groups are Fitting groups, J. Algebra 270 (2003), 7-27.

[21] Gunnar Traustason, Two generator 4-Engel groups, Internat. J. Algebra and Comput. (to appear).

[22] M.R. Vaughan-Lee, Engel-4 groups of exponent 5, Proc. London Math. Soc. 74 (1997), 306-334. 